\title{
3D Analysis of the Synaptic Organization in the Entorhinal Cortex in Alzheimer's Disease
}

\author{
M. Domínguez-Álvaro, ${ }^{1}$ M. Montero-Crespo, ${ }^{1,2,3}$ L. Blazquez-Llorca, ${ }^{1,3,4}$ S. Plaza-Alonso, ${ }^{1,2,3}$ \\ N. Cano-Astorga, ${ }^{1,2,3}$ J. DeFelipe, ${ }^{1,2,3}$ and ${ }^{-}$L. Alonso-Nanclares ${ }^{1,2,3}$
}

https://doi.org/10.1523/ENEURO.0504-20.2021

${ }^{1}$ Laboratorio Cajal de Circuitos Corticales, Centro de Tecnología Biomédica, Universidad Politécnica de Madrid, Madrid 28223, Spain, ${ }^{2}$ Instituto Cajal, Consejo Superior de Investigaciones Científicas (CSIC), Madrid 28002, Spain, ${ }^{3}$ Centro de Investigación Biomédica en Red sobre Enfermedades Neurodegenerativas (CIBERNED), Instituto de Salud Carlos III 28031, Madrid, Spain, and ${ }^{4}$ Sección Departamental de Anatomía y Embriología (Veterinaria), Facultad de Veterinaria, Universidad Complutense de Madrid, Madrid 28040, Spain

\begin{abstract}
The entorhinal cortex (EC) is especially vulnerable in the early stages of Alzheimer's disease (AD). In particular, cognitive deficits have been linked to alterations in the upper layers of EC. In the present report, we examined Layers II and III from eight human brain autopsies (four subjects with no recorded neurologic alterations and four $A D$ cases). We used stereological methods to assess cortical atrophy of the EC and possible changes in the volume occupied by different cortical elements (neuronal and glial cell bodies; blood vessels; and neuropil). We performed 3D ultrastructural analyses of synapses using focused ion beam/scanning electron microscopy (FIB/SEM) to examine possible alterations related to AD. At the light microscope level, we found a significantly lower volume fraction occupied by neuronal bodies in Layer III and a higher volume fraction occupied by glial cell bodies in Layer II in AD cases. At the ultrastructural level, we observed that (1) there was a significantly lower synaptic density in both layers in AD cases; (2) synapses were larger and more complex in Layer II in AD cases; and (3) there was a greater proportion of small and simple synapses in Layer III in AD cases than in control individuals. These structural differences may play a role in the anatomic basis for the impairment of cognitive functions in AD.
\end{abstract}

Key words: dementia; electron microscopy; FIB/SEM; medial temporal lobe; neuropil; postsynaptic targets

\section{Significance Statement}

Analysis of the synaptic characteristics provides critical data on synaptic organization. Using 3D electron microscopy (EM), the present study shows the synaptic organization of the neuropil of the human entorhinal cortex (EC) at the ultrastructural level. The EC is especially vulnerable in the early stages of Alzheimer's disease (AD). Our present results show structural differences that may contribute as anatomic basis for the impairment of cognitive functions in $A D$. Thus, these results may help to understand the relationship between alterations of the synaptic circuits and the cognitive deterioration in AD.

\section{Introduction}

Alzheimer's disease (AD) is a progressive neurodegenerative disease which is considered to be the main cause of dementia. The brain of AD cases shows brain atrophy and, at the neuropathological level, the most

Received November 23, 2020; accepted April 16, 2021; First published May 26, 2021.

The authors declare no competing financial interests. characteristic findings include the presence of extracellular amyloid- $\beta(\mathrm{A} \beta)$ plaques and intracellular neurofibrillary tangles (NFTs) of filamentous aggregates of hyperphosphorylated tau protein (Alzheimer's Association, 2020). $\mathrm{A} \beta$ plaques and NFTs are mostly found in the cerebral

Author contributions: J.D. and L.A.-N. designed research; M.D.-Á., M.M.-C., L.B.-L., and L.A.-N. performed research; M.D.-Á., S.P.-A., N.C.-A., and L.A.-N. analyzed data; M.D.-Á., M.M.-C., L.B.-L., S.P.-A., N.C.-A., J.D., and L.A.-N. wrote the paper. 
cortex, where both their numbers and the proportion of the cortex affected by them increase progressively as the disease advances (Braak and Braak, 1991; Dickson, 1997; Thal et al., 2002). Studies focusing on AD progression have shown that entorhinal cortex (EC) is one of the first brain regions affected by the presence of altered tau protein (Braak and Braak, 1991).

The EC has been shown to be essential for memory functions and spatial navigation (for review, see Schultz et al., 2015), and alterations in its upper layers have been related to cognitive deficits in AD individuals (Van Hoesen et al., 1991; Gómez-Isla et al., 1996). EC is considered to be an interface between the hippocampal formation and a large variety of association and limbic cortices (Solodkin and Van Hoesen, 1996; Lavenex and Amaral, 2000). In particular, the EC is the origin of the perforant pathway (from Layers II and III), which provides the largest input source to the hippocampal formation, targeting the ammonic fields (CA) CA1, CA2, and CA3, as well as the dentate gyrus (DG) and subiculum. Specifically, Layer II neurons project to CA1 via the trisynaptic circuit, passing through the DG and CA3, while Layer III neurons project directly to CA1 in the monosynaptic pathway (Insausti and Amaral, 2012; Kondo et al., 2009). It has been proposed that the trisynaptic pathway is more susceptible to premature degeneration (for review, see Van Hoesen et al., 2006; Llorens-Martín et al., 2014).

The presence of pathologic forms of $A \beta$ and tau proteins in the cerebral cortex of $A D$ cases has been related to neuronal loss, synapse alterations and dendritic spine degeneration (for review, see Forner et al., 2017; Chen et al., 2019). The loss and dysfunction of synapses have been proposed as the major structural correlates of the cognitive decline associated with AD (Coleman et al., 2004; Dickson et al., 1995; Selkoe, 2002; Sze et al., 1997). It has been proposed that synaptic loss affects the subcortical regions and the EC first and then progresses to other cortical regions (Braak and Braak, 1991; Braak and Del Tredici, 2012, 2020). Therefore, deciphering the changes that affect the normal function of synapses may

This work was supported by the Ministerio de Ciencia e Innovación Grant PGC2018-094307-B-100, the Centro de Investigación Biomédica en Red sobre Enfermedades Neurodegenerativas (CIBERNED, Spain) Grant CB06/05/0066), the Alzheimer's Association Grant ZEN-15-321663, and the European Union's Horizon 2020 Framework Programme for Research and Innovation Grant 945539 (Human Brain Project SGA3). L.B.-L. gained a postdoctoral contract from the Universidad Nacional de Educación a Distancia (UNED) (Plan de Promoción de la Investigación, 2014-040-UNED-POST). M.M.-C. was supported by the Spanish Ministerio de Educación y Formación Profesional Research Fellowship FPU14/02245. N.C.-A. was supported by the Spanish Ministerio de Ciencia e Innovación Research Fellowship PRE2019-089228. S. P.-A. was supported by the Spanish Ministerio de Ciencia e Innovación Research Fellowship FPU19/00007.

Acknowledgements: We thank Carmen Álvarez and Lorena Valdés for their technical assistance and Nick Guthrie for his excellent text editing.

Correspondence should be addressed to L. Alonso-Nanclares at aidi@ cajal.csic.es.

https://doi.org/10.1523/ENEURO.0504-20.2021

Copyright (๑) 2021 Domínguez-Álvaro et al.

This is an open-access article distributed under the terms of the Creative Commons Attribution 4.0 International license, which permits unrestricted use, distribution and reproduction in any medium provided that the original work is properly attributed. contribute to better understanding of the pathologic mechanisms of $A D$.

In the present study, we performed 3D ultrastructural analysis of the EC using focused ion beam/scanning electron microscopy (FIB/SEM) and specific software that allows the segmentation of synapses in a reconstructed 3D volume (Morales et al., 2011). This technology greatly facilitates the analysis of possible alterations at the synaptic level in $A D$, as previously shown in the human transentorhinal cortex and the CA1 hippocampal region (Domínguez-Álvaro et al., 2019; Montero-Crespo et al., 2021). Our goal was to investigate the possible synaptic changes occurring in Layers II and III of the EC related to $A D$, not only with regard to numbers, spatial distribution and types of synapses, but also regarding the morphologic characteristics of each synapse (shape and size), as well as possible changes in their postsynaptic targets. For this purpose, we performed an analysis of the neuropil from Layers II and III of the EC, from eight human brain autopsies (four subjects with no recorded neurologic alterations and four $A D$ cases) with short postmortem delays $(<3.5 \mathrm{~h})$. Since the presence of $\mathrm{A} \beta$ plaques is related to a virtual lack of synapses in their vicinity (Blazquez-Llorca et al., 2013), we focused on the neuropil that was free of plaques. We also used stereological methods to assess cortical atrophy of the EC, and possible changes in the volume occupied by different cortical elements (neuronal and glial cell bodies, blood vessels and neuropil) at the light microscope level.

\section{Materials and Methods}

\section{Tissue preparation}

Human brain tissue was obtained from eight autopsies (four male and four female subjects) with short postmortem delays $(<3.5 \mathrm{~h}$; supplied by Instituto de Neuropatología del IDIBELL-Hospital Universitario de Bellvitge, Barcelona, Spain; Unidad Asociada Neuromax, Laboratorio de Neuroanatomía Humana, Facultad de Medicina, Universidad de Castilla-La Mancha, Albacete and the Laboratorio Cajal de Circuitos Corticales, Universidad Politécnica de MadridConsejo Superior de Investigaciones Científicas). The sampling procedure was approved by the Institutional Ethical Committees of each of the institutions involved. Tissue from some of these human brains has been used in previous studies (Domínguez-Álvaro et al., 2018, 2019, 2021; MonteroCrespo et al., 2020, 2021; Cano-Astorga et al., 2021).

Briefly, tissue samples were obtained from four control cases (non-demented subjects with no recorded neurologic or psychiatric alterations) and four AD cases according to the neuropathological criteria provided by the above-mentioned centers (Table 1; Mirra et al., 1991).

Upon removal, the brain tissue was fixed in cold $4 \%$ paraformaldehyde (Sigma-Aldrich) in $0.1 \mathrm{M}$ sodium phosphate buffer (PB; Panreac, 131965), pH 7.4, for 24-48 h. After fixation, the tissue was washed in PB and coronally sectioned ( $150 \mu \mathrm{m}$ thick) in a vibratome (Vibratome Sectioning System, VT1200S Vibratome, Leica Biosystems). Sections containing EC were selected for Nissl staining, immunohistochemistry, and EM processing (Fig. 1). 
Table 1: Clinical and neuropathological information

\begin{tabular}{|c|c|c|c|c|c|c|c|}
\hline Case & Sex & $\begin{array}{l}\text { Age } \\
\text { (years) }\end{array}$ & Cause of death & $\begin{array}{l}\text { Postmortem } \\
\text { delay }(\mathrm{h})\end{array}$ & $\begin{array}{l}\text { Braak } \\
\text { stage }\end{array}$ & $\begin{array}{l}\text { CERAD } \\
\text { stage }\end{array}$ & Neuropsychological diagnosis \\
\hline AB3 & Male & 53 & Bladder carcinoma & 3.5 & 0 & 0 & No recorded neurologic alterations \\
\hline M16 & Male & 40 & Traffic accident & 3 & 0 & 0 & No recorded neurologic alterations \\
\hline IF1 & Female & 80 & - & 2 & IV & B & $\begin{array}{l}\text { No evidence of cognitive } \\
\text { impairment and dementia }\end{array}$ \\
\hline VK16 & Female & 88 & - & 2.5 & VI & C & Dementia \\
\hline VK22 & Female & 86 & - & 2 & V & C & Dementia \\
\hline
\end{tabular}

Braak stages (Braak and Braak, 1991): III, NFTs in EC and closely related areas; III-IV, NFTs abundant in amygdala and hippocampus; extending slightly into association cortex; V-VI, NFTs widely distributed throughout the neocortex and ultimately involving primary motor and sensory areas. CERAD stages (Mirra et al., 1991): A, low density of neuritic plaques; B, intermediate density of neuritic plaques; C, high density of neuritic plaques. -, not available.

Coronal sections of the human EC at medial level were used for the present study (for review, see Insausti et al., 2017). The delimitation of the EC was established by combining the Nissl and anti-NeuN markers (Fig. 1). The main cytoarchitectural characteristic of the EC that allows it to be recognized is the presence of large islands of modified pyramidal neurons and stellate cells in Layer II (Braak and Braak, 1992; Insausti and Amaral, 2012; Kobro-Flatmoen and Witter, 2019). Sections containing EC were selected for Nissl staining, immunohistochemistry and EM processing (Fig. 1).

\section{Immunohistochemistry}

The selected sections were first rinsed in $0.1 \mathrm{M} P B$, pretreated in $2 \% \mathrm{H}_{2} \mathrm{O}_{2}$ for 30 min to remove endogenous peroxidase activity, and then incubated for $1 \mathrm{~h}$ at room temperature in a solution of $3 \%$ normal horse serum (for monoclonal antibodies; Vector Laboratories Inc.) and $0.25 \%$ Triton X-100 (Merck). The sections were then incubated for $48 \mathrm{~h}$ at $4^{\circ} \mathrm{C}$ in the same solution with mouse anti-NeuN (1:2000; Chemicon; MAB377) and anti-human PHF $_{-T a u}$ antibody clone AT8 (1:2000, MN1020, Thermo Scientific); for the sake of clarity, we will refer to this as antiPHF $_{\text {-Tau-AT8. The sections selected for anti-A } \beta \text { were first }}$ treated with $88 \%$ formic acid (Sigma-Aldrich, \#251364) to ensure specific plaque immunostaining, and were then incubated in a solution containing mouse antibody anti-A $\beta$ (clone 6F/3D; 1:50, Dako M0872). The sections were then processed with a secondary biotinylated horse anti-mouse IgG antibody (1:200, Vector Laboratories), and then incubated for $1 \mathrm{~h}$ in an avidin-biotin peroxidase complex (Vectastain ABC Elite PK6100, Vector) and, finally, with the chromogen 3,3'-diaminobenzidine tetrahydrochloride (DAB; Sigma-Aldrich). Finally, the sections were dehydrated, cleared with xylene, and cover-slipped.

\section{Tissue processing for EM}

EC sections were postfixed for $24 \mathrm{~h}$ in a solution containing $2 \%$ paraformaldehyde, $2.5 \%$ glutaraldehyde (TAAB, G002) and 0.003\% $\mathrm{CaCl}_{2}$ (Sigma, C-2661-500G) in sodium cacodylate (Sigma, C0250-500G) buffer (0.1 M). These sections were washed in sodium cacodylate buffer
$(0.1 \mathrm{~m})$ and treated with $1 \% \mathrm{OsO}_{4}$ (Sigma, 05500), $0.1 \%$ potassium ferrocyanide (Probus, 23345) and $0.003 \%$ $\mathrm{CaCl}_{2}$ in sodium cacodylate buffer $(0.1 \mathrm{M})$ for $1 \mathrm{~h}$ at room temperature. After washing in $\mathrm{PB}$, the sections were stained with $2 \%$ uranyl acetate (EMS, 8473), and then dehydrated and flat-embedded in Araldite (TAAB, E021) for $48 \mathrm{~h}$ at $60^{\circ} \mathrm{C}$ (DeFelipe and Fairén, 1993). Embedded sections were glued onto a blank Araldite block and trimmed. Semithin sections (1-2 $\mu \mathrm{m}$ thick) were obtained from the surface of the block and stained with $1 \%$ toluidine blue (Merck, 115930) in 1\% sodium borate (Panreac, 141644).

The last semithin section (which corresponds to the section immediately adjacent to the block surface) was examined under light microscope and photographed to accurately locate the neuropil regions to be examined (Fig. 2). The blocks containing the embedded tissue were then glued onto a sample stub using conductive adhesive tabs (EMS 77825-09). All the surfaces of the block, except for the one to be studied (the top surface), were covered with silver paint (EMS 12630) to prevent charging artifacts. The stubs with the mounted blocks were then placed into a sputter coater (Emitech K575X, Quorum Emitech) and the top surface was coated with a 10- to 20$\mathrm{nm}$-thick layer of gold/palladium to facilitate charge dissipation.

\section{Cortical thickness estimation}

In order to estimate the atrophy of the EC, we measured the cortical thickness in three to five toluidine blue-stained semithin sections from each of the cases, obtained in the coronal plane of the cortex and containing the entire cortex, tracing a perpendicular line from the pial surface to the white matter. Measurements of the distance between the pial surface and the boundary with the white matter were performed using Fiji program (ImageJ 1.51; National Institutes of Health; http://imagej.nih.gov/ij/). To average data, three measurements were made per section.

\section{Volume fraction estimation of cortical elements}

Three to five semithin sections (1-2 $\mu \mathrm{m}$ thick) stained with $1 \%$ toluidine blue were used to estimate 
A

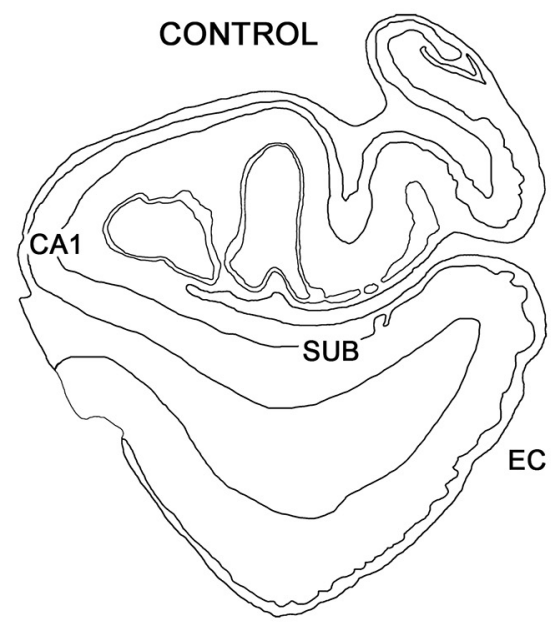

B

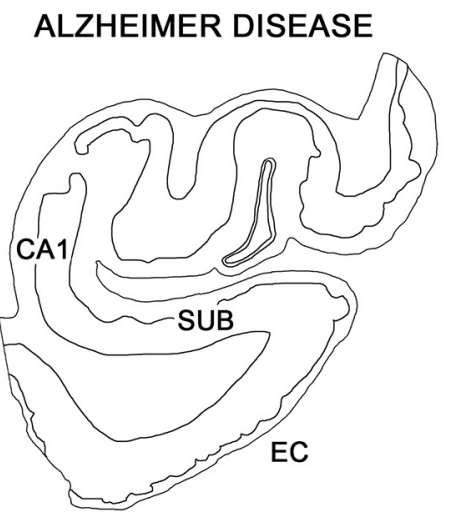

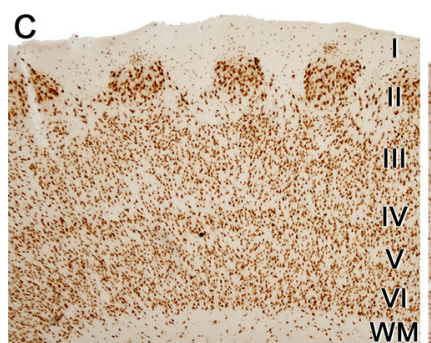

$\mathrm{F}$

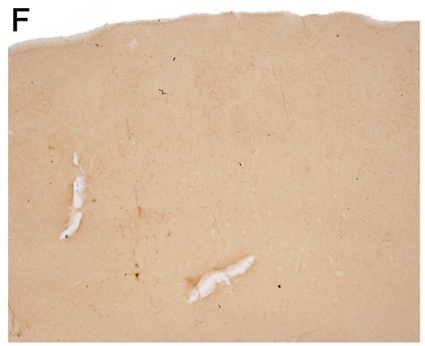

I

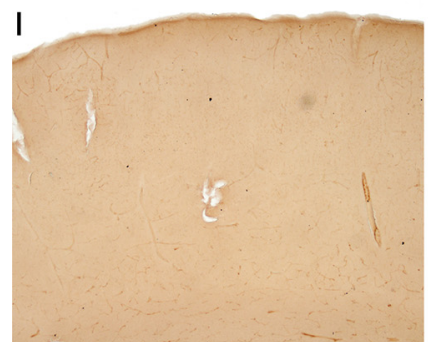

D

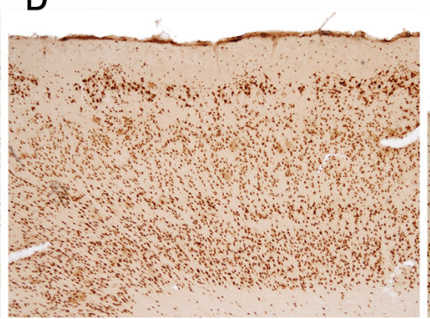

G

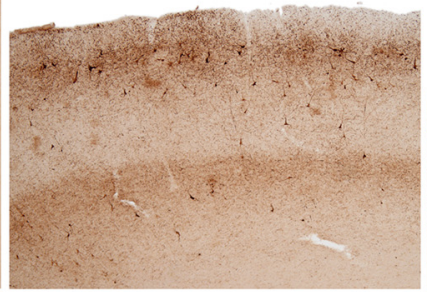

$J$

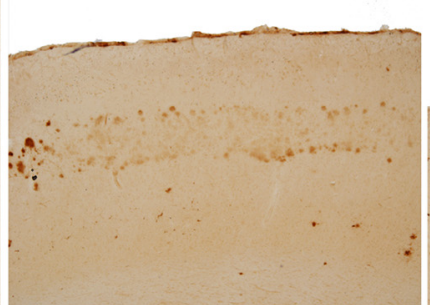

E

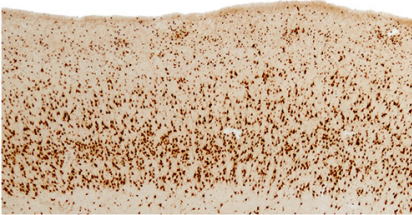

$\mathrm{H}$

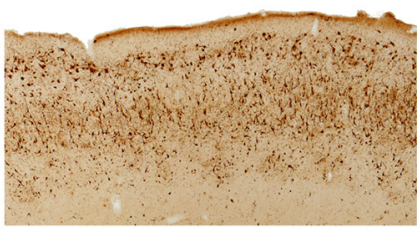

$\mathrm{K}$

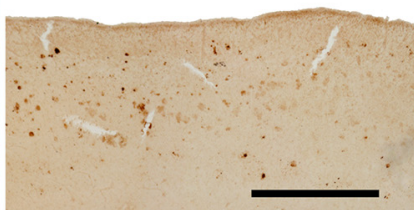

Figure 1. $E C$ of control and $A D$ cases. Plots of the medial structures including the hippocampal formation and EC from control $(\boldsymbol{A})$ and $A D(B)$ cases to illustrate the atrophy that occurs in AD. Plots are based on Nissl-stained coronal sections from cases AB3 and IF1. Microphotographs showing the EC in a control case AB3 $(\boldsymbol{C}, \boldsymbol{F}, \boldsymbol{I})$ and in AD cases IF1 $(\boldsymbol{D}, \boldsymbol{G}, \boldsymbol{J})$ and VK16 $(\boldsymbol{E}, \boldsymbol{H}, \boldsymbol{K})$. Layers are indicated to the right of panel $\boldsymbol{C}$. Sections are immunostained with antibodies anti-NeuN $(\boldsymbol{C}-\boldsymbol{E})$, anti-PHF-Tau-AT8 $(\boldsymbol{F}-\boldsymbol{H})$, and anti$\mathrm{A} \beta(\boldsymbol{I}-\boldsymbol{K})$. Note the intense labeling of PHF-Tau-AT8-positive neurons $(\boldsymbol{G}, \boldsymbol{H})$ and A $\beta$-positive plaques $(\boldsymbol{J}, \boldsymbol{K})$ in $A D$ cases, particularly in case VK16, and the lack of labeling in the control case. CA1, cornu ammonis field 1; SUB, subiculum; WM, white matter. Scale bar in $\boldsymbol{K}: 4 \mathrm{~mm}(\boldsymbol{A}, \boldsymbol{B})$ and $1 \mathrm{~mm}(\boldsymbol{C}-\boldsymbol{K})$.

the respective volume fractions $(\mathrm{Vv})$ occupied by blood vessels, cell bodies (glia and neurons) and neuropil. This estimation was performed applying the Cavalieri principle (Gundersen et al., 1988) by point counting using the integrated Stereo Investigator stereological package (version 8.0, MicroBright-
Field Inc.) attached to an Olympus light microscope (Olympus) at $40 \times$ magnification. A grid, whose points covered an area of $400 \mu \mathrm{m}^{2}$, was overlaid over each semithin section to determine the $V_{v}$ occupied by the different elements: neurons, glia, blood vessels, and neuropil. 

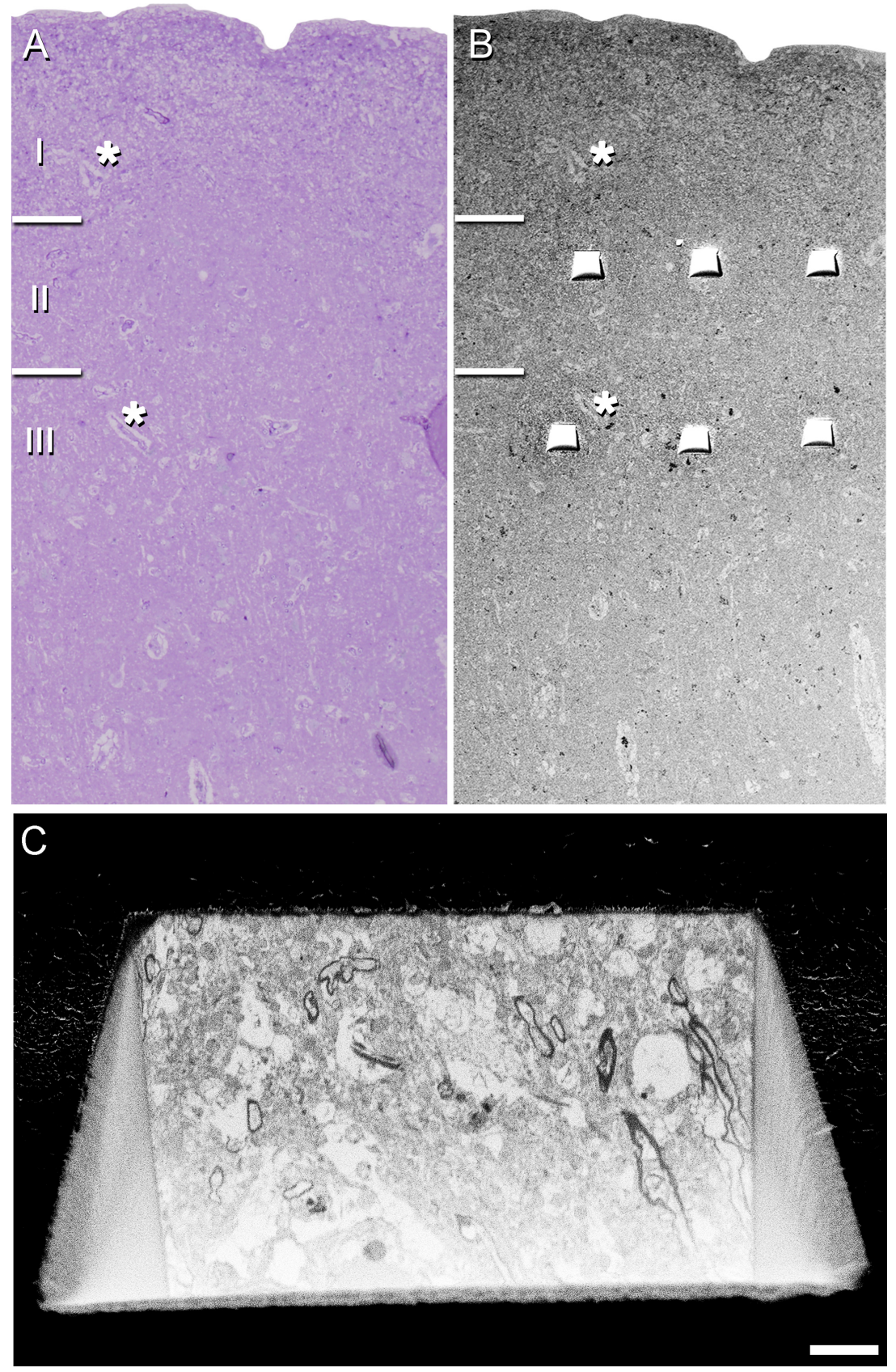

Figure 2. Correlative light/EM of Layers II and III of the EC. Delimitation of layers is based on the staining pattern of 1- $\mu$ m-thick semithin section, stained with toluidine blue $(\boldsymbol{A})$, which is adjacent to the block for FIB/SEM imaging (B). $\boldsymbol{B}$, SEM image illustrating the block surface with trenches made in the neuropil (three per layer). Asterisks in $\boldsymbol{A}, \boldsymbol{B}$ point to the same blood vessels, showing that the exact location of the region of interest was accurately determined. $\boldsymbol{C}$, SEM image showing the front of a trench made to expose the tissue and to acquire the FIB/SEM stack of images at the final magnification. Scale bar in $\boldsymbol{C}: 70 \mu \mathrm{m}(\boldsymbol{A}), 85 \mu \mathrm{m}(\boldsymbol{B})$, and $3 \mu \mathrm{m}(\boldsymbol{C})$.

\section{D EM}

The Araldite block containing the tissue was used to obtain images stacks from the EC (Fig. 2) using a dual beam microscope (FIB/SEM; Crossbeam 540 EM, Carl Zeiss NTS GmbH).

The dual beam microscope combines a high-resolution field-emission SEM column with a focused gallium ion beam (FIB), which permits removal of thin layers of material from the sample surface on a nanometer scale. As soon as one layer of material $(20 \mathrm{~nm}$ thick) is removed by the FIB, the exposed surface of the sample is imaged by the SEM using a backscattered electron detector. The sequential automated use of FIB milling and SEM imaging allowed us to obtain long series of photographs of a 3D sample of selected regions (Merchán-Pérez et al., 2009). FIB/SEM images from the neuropil were obtained avoiding the neuronal and glial somata, blood vessels and also avoiding $\mathrm{A} \beta$ plaques to eliminate the effect of alterations 
Table 2: Accumulated data obtained from the ultrastructural analysis of the neuropil from Layers II and III of the EC

\begin{tabular}{|c|c|c|c|c|c|c|c|c|c|c|c|}
\hline $\begin{array}{l}\mathrm{EC} \\
\text { layer } \\
\end{array}$ & Group & $\begin{array}{l}\text { No. } \\
\text { AS }\end{array}$ & $\begin{array}{l}\text { No. } \\
\text { SS }\end{array}$ & $\begin{array}{l}\text { No. all } \\
\text { synapses }\end{array}$ & $\begin{array}{l}\% \text { AS } \\
(\text { mean } \pm \mathrm{SD})\end{array}$ & $\begin{array}{l}\% \text { SS } \\
(\text { mean } \pm \text { SD) }\end{array}$ & $\begin{array}{l}\text { CF volume } \\
\left(\mu \mathrm{m}^{3}\right)\end{array}$ & $\begin{array}{l}\text { No. } A S / \mu \mathrm{m}^{3} \\
\text { (mean } \pm \mathrm{SD})\end{array}$ & $\begin{array}{l}\text { No. SS } / \mu \mathrm{m}^{3} \\
\text { (mean } \pm \mathrm{SD} \text { ) }\end{array}$ & $\begin{array}{l}\text { No. all } \\
\text { synapses } / \mu \mathrm{m}^{3} \\
\text { (mean } \pm \mathrm{SD} \text { ) }\end{array}$ & $\begin{array}{l}\text { Distance to } \\
\text { nearest } \\
\text { neighbor } \\
(\mathrm{nm} \text {; mean } \pm \mathrm{SD})\end{array}$ \\
\hline \multirow[t]{2}{*}{ II } & Control & 1553 & 137 & 1690 & $91.90 \pm 2.62$ & $8.10 \pm 2.62$ & $4221(5445)$ & $0.37 \pm 0.05(0.38 \pm 0.06)$ & $0.03 \pm 0.01(0.03 \pm 0.01)$ & $0.40 \pm 0.03(0.41 \pm 0.06)$ & $840 \pm 35(811 \pm 33)$ \\
\hline & Alzheimer & 954 & 90 & 1044 & $90.53 \pm 4.27$ & $9.47 \pm 4.27$ & 4409 (5742) & $0.21 \pm 0.09(0.23 \pm 0.09)$ & $0.02 \pm 0.01(0.02 \pm 0.01)$ & $0.24 \pm 0.09(0.25 \pm 0.09)$ & $938 \pm 113(906 \pm 109)$ \\
\hline \multirow[t]{2}{*}{ III } & Control & 1747 & 130 & 1877 & $92.84 \pm 1.97$ & $7.16 \pm 1.978$ & $4371(5466)$ & $0.40 \pm 0.09(0.42 \pm 0.09)$ & $0.03 \pm 0.01(0.03 \pm 0.01)$ & $0.43 \pm 0.01(0.45 \pm 0.09)$ & $852 \pm 42(823 \pm 41)$ \\
\hline & Alzheimer & 973 & 92 & 1065 & $92.12 \pm 5.70$ & $7.88 \pm 5.70$ & $4597(5653)$ & $0.22 \pm 0.06(0.23 \pm 0.08)$ & $0.02 \pm 0.02(0.02 \pm 0.02)$ & $0.24 \pm 0.08(0.25 \pm 0.09)$ & $1004 \pm 214(970 \pm 206)$ \\
\hline
\end{tabular}

All volume data are corrected for shrinkage factor. Data in parentheses are not corrected with the shrinkage and the fixation artifact factors. The data for individual cases are shown in Extended Data Table 2-1.

of synapses in the vicinity of $A \beta$-plaques, which has been described previously (Blazquez-Llorca et al., 2013).

In total, 24 stacks of images of the neuropil from Layers II and III of the EC from AD cases were obtained (three stacks for each of the 4 cases, with a total volume studied of $9006 \mu \mathrm{m}^{3}$; Table 2). The data obtained from these stacks of images were compared with the data obtained in the same regions of four control cases (DomínguezÁlvaro et al., 2021).

\section{Synaptic 3D analysis}

FIB/SEM stacks of images were analyzed using EspINA software (EspINA Interactive Neuron Analyzer, 2.1.9; https://cajalbbp.es/espina/), which allows the segmentation of synapses in the reconstructed 3D volume (Morales et al., 2011; Fig. 3). Since the EspINA software allows navigation through the stack of images (Figs. 3, 4; Movies 1, 2 ), it was possible to unambiguously identify every synapse as asymmetric synapses (AS) or symmetric synapses (SS), based on the thickness of the PSD (Fig. 4). There
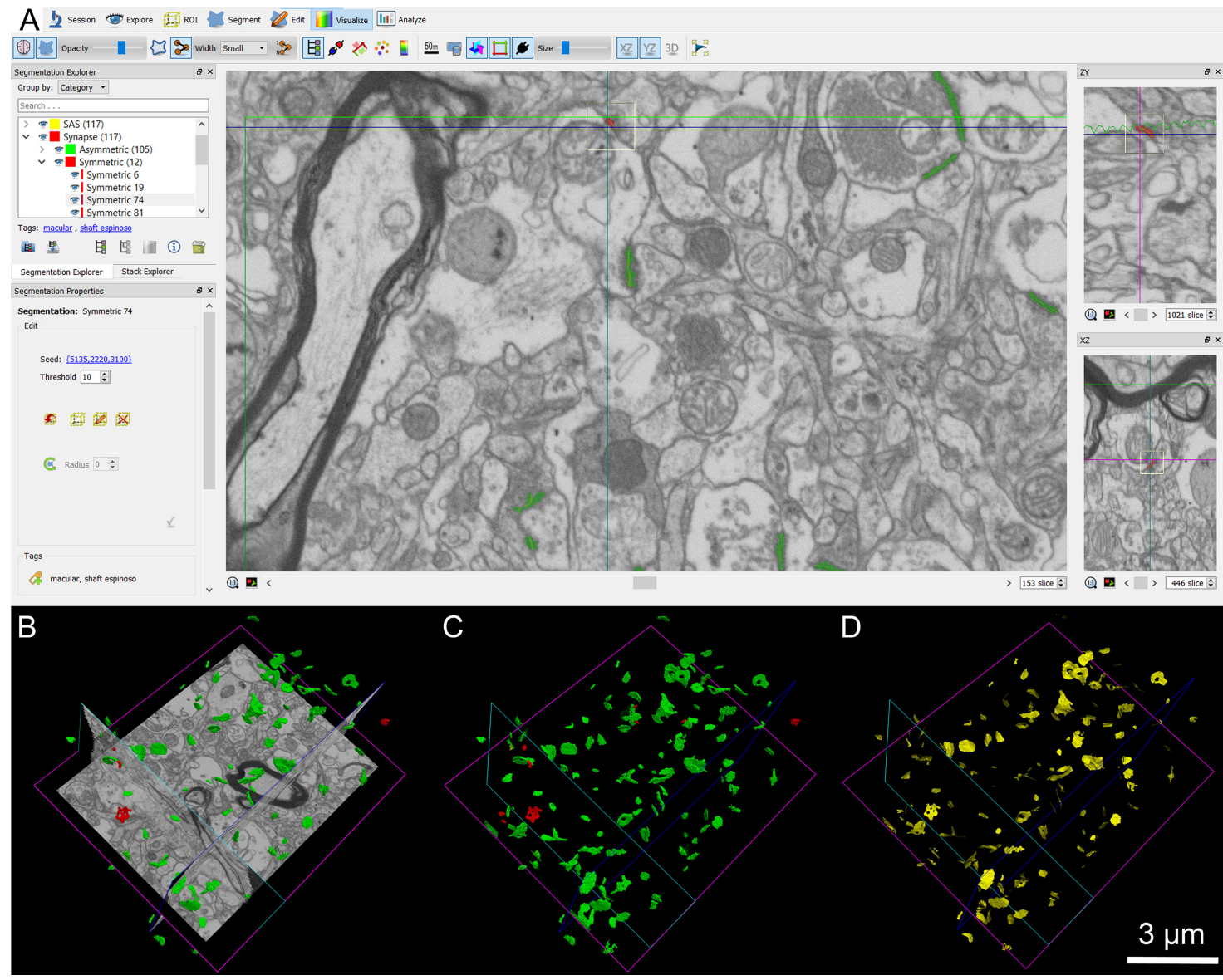

Figure 3. Screenshot of the EspINA software user interface. $\boldsymbol{A}$, In the main window, the sections are viewed through the $x y$ plane (as obtained by FIB/SEM microscopy). The other two orthogonal planes, $y z$ and $x z$, are also shown in adjacent windows on the right. $\boldsymbol{B}$. The 3D windows show the three orthogonal planes and the 3D reconstruction of AS (green) and SS (red) segmented synapses, the reconstructed synapses $(\boldsymbol{C})$, and the computed SAS for each reconstructed synapse (in yellow; $\boldsymbol{D})$. 

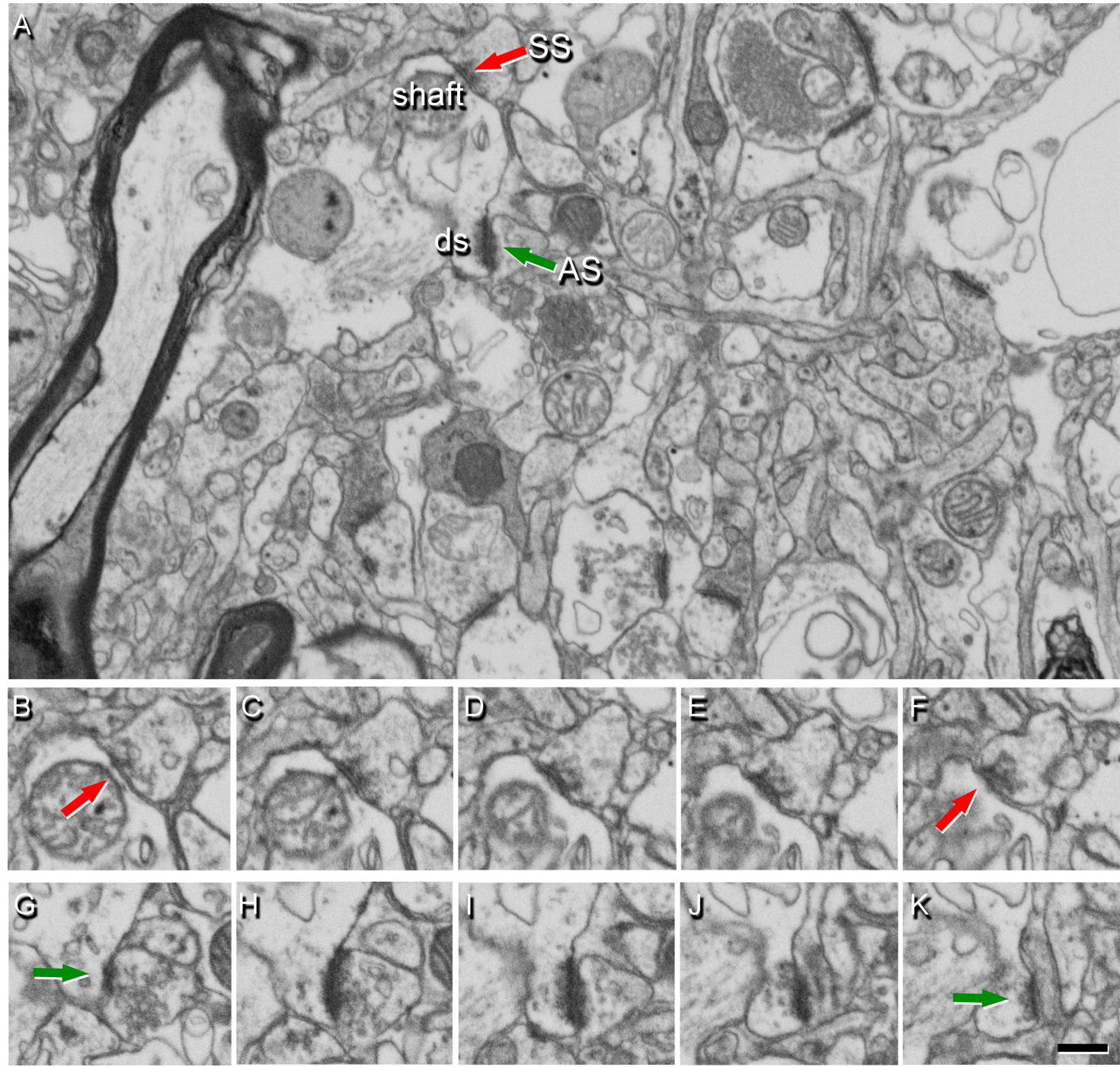

Figure 4. Identification of synapses in serial EM images obtained by FIB/SEM. $\boldsymbol{A}$, Image from Layer II showing the neuropil from an AD case, with two synapses indicated (arrows) as examples of SS (red) on a dendritic shaft, and AS (green) on a dendritic spine head (ds). Synapse classification was based on examination of the full sequence of serial images; the SS can be visualized in $\boldsymbol{B}-\boldsymbol{F}$, and the AS in $\mathbf{G}-\boldsymbol{K}$. Scale bar in $\boldsymbol{K}: 460 \mathrm{~nm}(\boldsymbol{A})$ and $360 \mathrm{~nm}(\boldsymbol{B}-\boldsymbol{K})$.

is a consensus for classifying cortical synapses into AS (or Type I) and SS (or Type II). The main characteristic distinguishing these synapses is the prominent or thin postsynaptic density, respectively: synapses with prominent PSDs are classified as AS, while thin PSDs are classified as SS (Gray, 1959; Peters and Palay, 1996).

EspINA also allowed the application of an unbiased 3D counting frame (CF) to obtain the synaptic density per volume (for details, see Merchán-Pérez et al., 2009). The synaptic density values were obtained by dividing the total number of synapses by the total volume of the CF. Geometrical characteristics, such as size, and spatial distribution features (centroids) of each reconstructed synapse were also calculated by EspINA.

EspINA software extracts the synaptic apposition surface (SAS) and provides its morphologic measurements (Fig. 3). Since the presynaptic and postsynaptic densities are located face to face, their surface areas are comparable (for details, see Morales et al., 2013). Since the SAS comprises both the active zone and the PSD, it is a functionally relevant measure of the size of a synapse (Morales et al., 2013). In addition, the visualization of each $3 \mathrm{D}$ reconstructed synapse allowed us to determine the synaptic morphology. Based on the presence of perforations or indentations in their perimeters, the synapses could be classified into four types: macular (with a flat, disk-shaped PSD), perforated (with one or more holes in the PSD), horseshoe (with an indentation in the perimeter of the PSD) or fragmented (with two or more physically discontinuous PSDs; for detailed description, see Domínguez-Álvaro et al., 2019).

To identify the postsynaptic targets of the synapses, we navigated the image stack using EspINA to determine whether the postsynaptic element was a dendritic spine ("spine" or "spines," for simplicity) or a dendritic shaft (Fig. 5; Movies 1, 2). Unambiguous identification of spines 


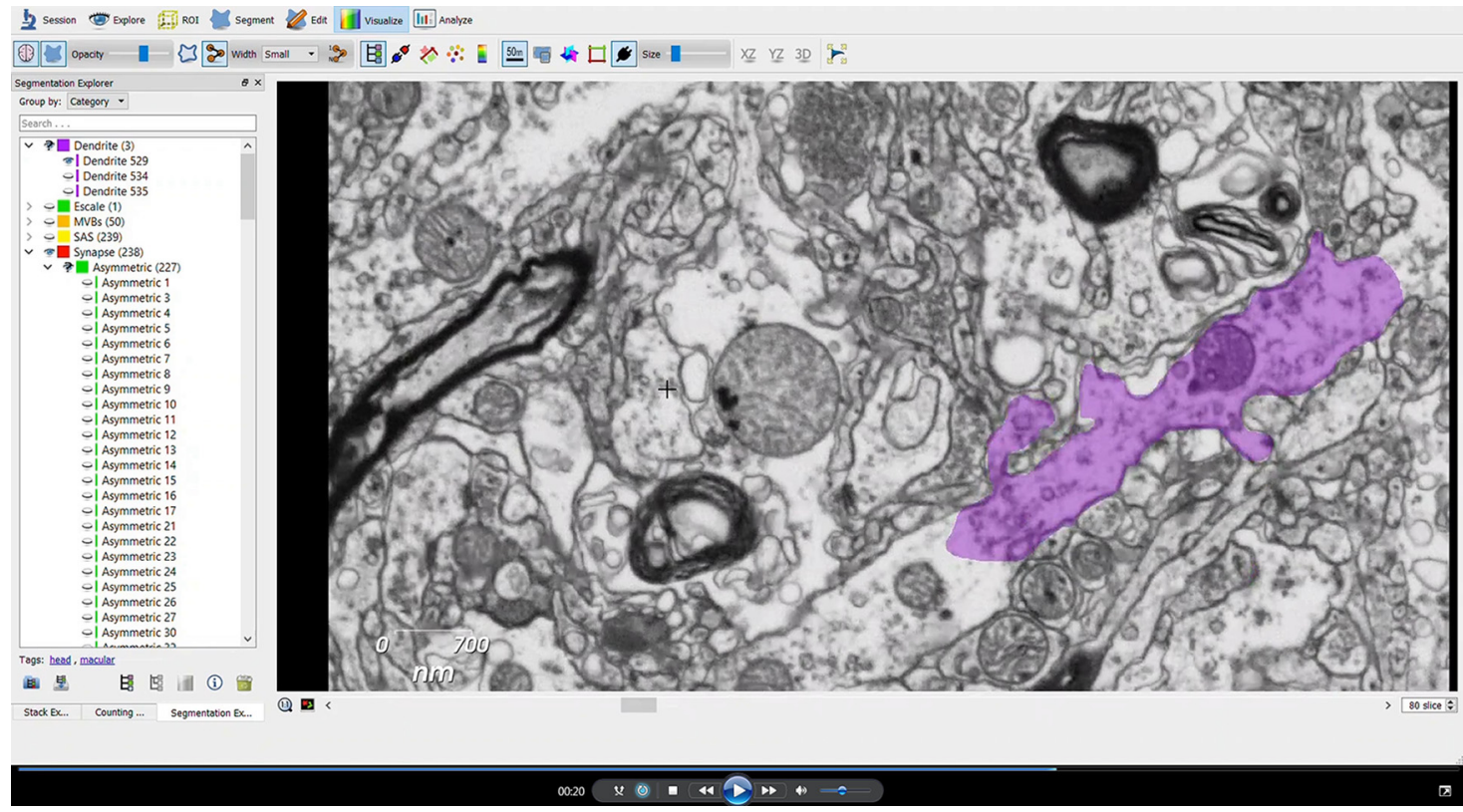

Movie 1. EspINA software user interface. FIB/SEM sections are viewed through the $x y z$ planes. AS (green) are shown, established on dendritic spines originating from a dendritic shaft (purple), shown as D529 in Figure 5. [View online]

as postsynaptic targets requires the spine to be visually traced to the parent dendrite, yielding what we refer to as fully reconstructed spines. Additionally, when synapses were established on a spine head-shaped postsynaptic element whose neck could not be followed to the parent dendrite, or whose neck was truncated, we identified these elements as non-fully reconstructed spines. These non-fully reconstructed spines were identified on the basis of their size and shape, the lack of mitochondria and the presence of a spine apparatus, or because they were filled with a characteristic fluffy material (used to describe the fine and indistinct filaments present in the spines), a term coined by Peters et al. (1991; see also del Río and DeFelipe, 1995). For simplicity, we will refer to the fully reconstructed and non-fully reconstructed spines as spines, unless otherwise specified. Similarly, for the unambiguous identification of dendritic shafts, it is necessary to be able to visually trace them inside the stack.
Accordingly, when the postsynaptic element of a synapse was close to the margins, and it was truncated by the borders of the stack, the identity of the postsynaptic target could not be determined. Therefore, the targets of synapses in each of the stacks were classified into two main categories: spines and dendritic shafts, while truncated elements that could not be safely identified were discarded. When the postsynaptic target was a spine, we further recorded the position of the synapse on the head or neck. Additionally, when the postsynaptic element was identified as a dendritic shaft, it was classified as "with spines" or "without spines."

\section{Spatial distribution analysis of synapses}

To analyze the spatial distribution of synapses, spatial point-pattern analysis was performed as described elsewhere (Merchán-Pérez et al., 2014). For each of the 24

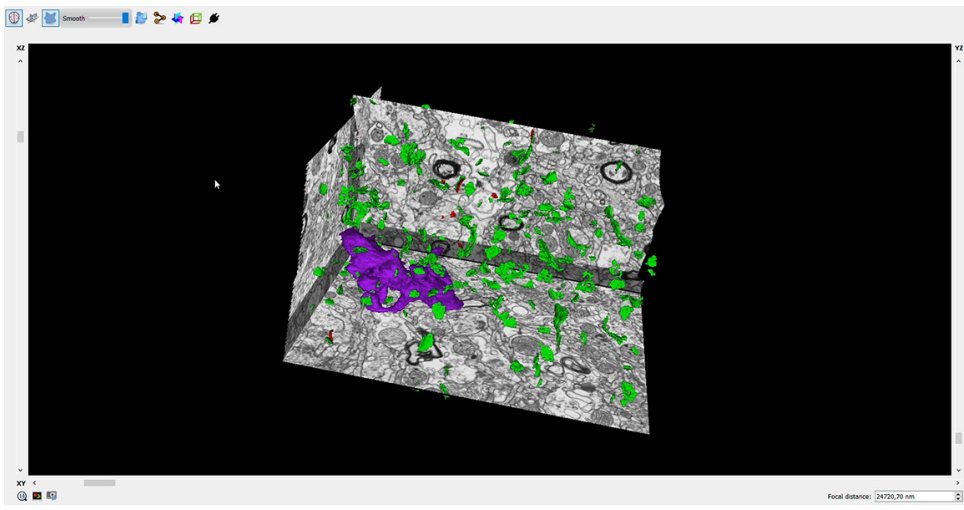

Movie 2. EspINA software 3D viewer. A stack of images is fully represented in the three orthogonal planes $(x, y$, and $z)$. The video shows the 3D reconstruction of a single dendritic segment (purple; D529 shown in Fig. 5) and the 3D reconstruction of all AS (green) and SS (red) established in this 3D reconstructed fragment. [View online] 


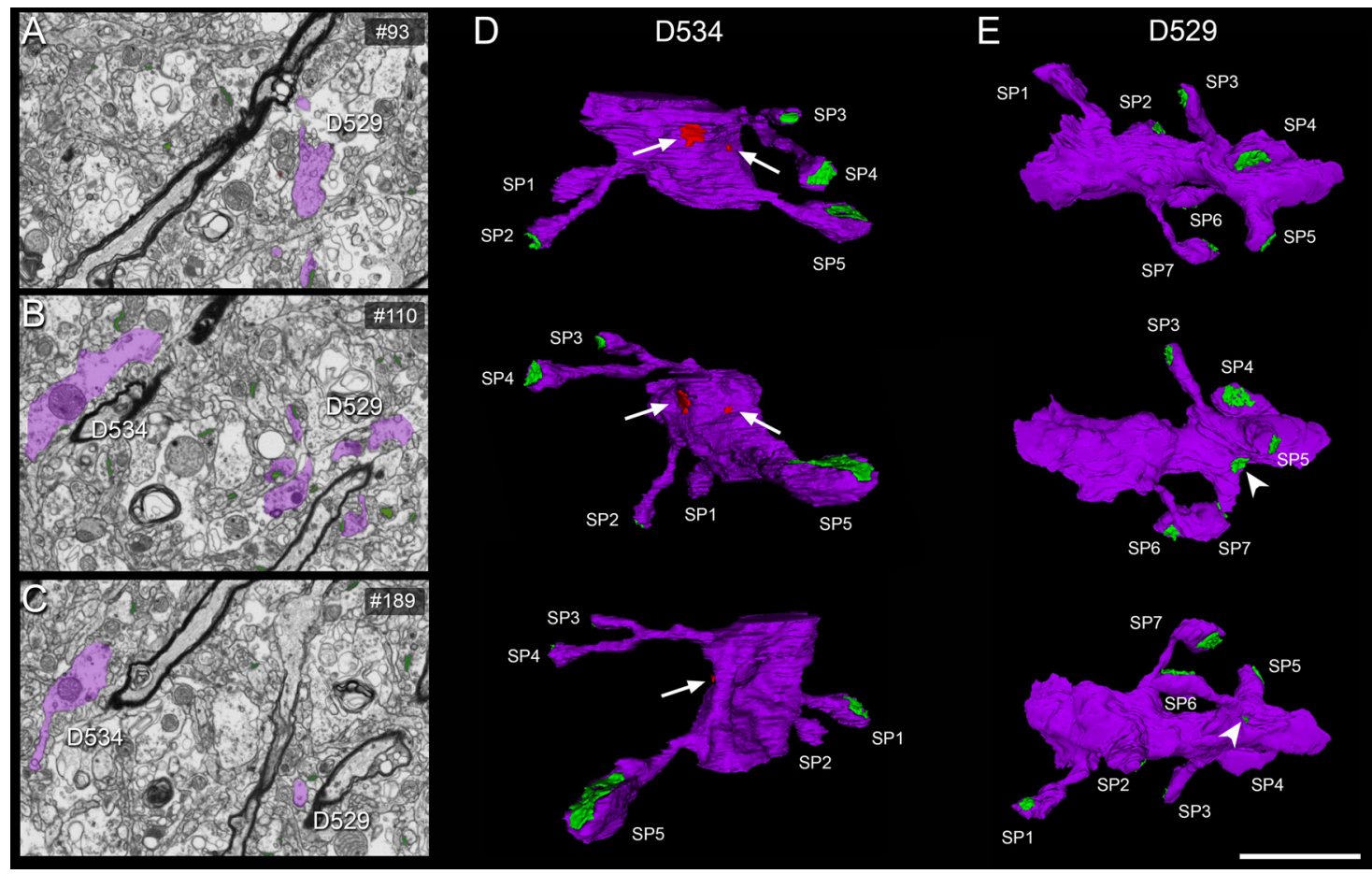

Figure 5. 3D reconstruction of dendritic segments from FIB/SEM serial images. A-C, Serial images showing two dendritic segments (D529, D534) partially reconstructed (in purple). 3D reconstructions of these dendritic segments are displayed in panels $\boldsymbol{D}, \boldsymbol{E}$. $\boldsymbol{D}, 3 \mathrm{D}$ reconstructed dendritic segment D534 in three views after rotation about the major dendritic axis. Five dendritic spines (SP 1-5) are shown, establishing AS (green), and two SS (arrows) on the shaft (red) are also visible. E, 3D reconstructed dendritic segment D534 in three views after rotation about the major dendritic axis. Seven dendritic spines (SP 1-7) establishing AS (green) are illustrated. Note that the synapse on SP4 can be identified as perforated in the middle panel $(\boldsymbol{E})$. The arrowhead indicates an AS on a spine neck. Scale bar in $\boldsymbol{E}$ : $3 \mu \mathrm{m}(\boldsymbol{A}-\boldsymbol{C})$ and $2 \mu \mathrm{m}(\boldsymbol{D}, \boldsymbol{E})$.

different samples, we calculated three functions commonly used for spatial point pattern analysis: F, G, and K functions (for detailed description, see Blazquez-Llorca et al., 2015). Additionally, we measured the distance of each synapse to its nearest synapse, to compare control and $A D$ samples. This study was conducted using the Spatstat package and R Project program (Baddeley et al., 2015).

\section{Tissue shrinkage}

All measurements were corrected for the tissue shrinkage that occurs during osmication and plastic-embedding of the vibratome sections containing the area of interest, as described previously (Merchán-Pérez et al., 2009). We measured the surface area and thickness of the vibratome sections with Stereo Investigator (MBF Bioscience), both before and after they were processed for EM (Oorschot et al., 1991). The surface area after processing was divided by the value before processing to obtain an area shrinkage factor $\left(p^{2}\right)$ of 0.933 . The linear shrinkage factor for measurements in the plane of the section $(p)$ was therefore 0.966 . The shrinkage factor in the $z$-axis was 0.901 . In addition, the total volume was corrected for the presence of fixation artifacts, which did not affect the accurate identification and quantitation of synapses (i.e., swollen neuronal or glial processes). The volume occupied by these artifacts was calculated applying the Cavalieri principle (Gundersen et al., 1988) and was discounted from the volume of the stacks of images to avoid underestimation of the number of synapses per volume. Specifically, a stereological grid with an associated area per point of $400,000 \mathrm{~nm}^{2}$ was superimposed onto each FIB/SEM stack using ImageJ Stereology Toolset (Mironov, 2017). Estimations were made every 20th section in each of the stacks. Every FIB/SEM stack was examined and the volume artifact ranged from $1 \%$ to $16 \%$ of the volume stacks. Volume fraction estimation was performed by point counting using the Cavalieri principle (Gundersen et al., 1988), in a similar fashion to the volume fraction estimation of cortical elements in semithin sections (see above, Volume fraction estimation of cortical elements).

All parameters measured were corrected to obtain an estimate of the preprocessing values. The shrinkage factor was used to correct the SAS area and perimeter data, while both the shrinkage and the fixation artifact factors were used to correct synaptic density values.

\section{Statistical analysis}

To determine possible differences between the control and $A D$ samples, statistical comparisons of the following parameters were conducted using the unpaired MannWhitney (MW) nonparametric $U$ test: cortical thickness, synaptic density, type of synapse (AS or SS), SAS size, 
synaptic shape, postsynaptic targets, and distance to the nearest neighboring synapse. To determine possible differences between control and AD samples regarding volume fractions (neuronal and glial cell bodies, blood vessels and neuropil), parametric $t$ test were conducted. Frequency distribution analyses were performed using Kolmogorov-Smirnov (KS) nonparametric test.

To perform statistical comparisons of AS and SS proportions, $\chi^{2}$ test was used for contingency tables. The same method was used to study whether there were significant differences between groups in relation to the shape of the synaptic junctions and their postsynaptic target. In all the $\chi^{2}$ statistical analyses, we firstly performed an "omnibus test" based on $2 \times 4$ contingency tables. To further investigate the specific cells driving the significance of the $\chi^{2}$ test, a partitioning procedure was applied to create $2 \times 2$ contingency tables (Sharpe, 2015).

Statistical analyses were performed using the GraphPad Prism statistical package (Prism 8.4.2 for Windows, GraphPad Software Inc.) and SPSS (IBM SPSS Statistics v24, IBM Corp.).

\section{Availability of data and materials}

Most data generated or analyzed during this study are included in the main text and the tables. The control datasets used and analyzed are available on the EBRAINS Knowledge Graph (doi: 10.25493/3GMJ-FEZ). Datasets used during the current study available from the corresponding author on reasonable request.

\section{Results}

Data regarding the synaptic organization of the human Layers II and III from the EC in the four control subjects has been previously published and detailed information can be found therein (Domínguez-Álvaro et al., 2021). What follows are the alterations of synapses in AD cases in comparison with control cases.

\section{Cortical thickness}

Measurements of the cortical thickness were derived from semi thin toluidine blue (serial sections) using light microscopy. The mean cortical thickness of the EC was $2.0 \pm 0.17 \mathrm{~mm}$ (mean $\pm \mathrm{SD}$ ) in the control group and $1.6 \pm 0.49 \mathrm{~mm}$ in the cases with AD. Although $\sim 20 \%$ lower EC cortical thickness in AD cases was observed, we did not find a statistically significant difference between the two groups (MW, $p=0.34$; Extended Data Table 1-1).

\section{Volume fraction of cortical elements}

In the control group Layer II, the values of the estimated Vv occupied by neuronal somata, glial somata, blood vessels, and neuropil were $4.5 \%, 2.6 \%, 3.8 \%$, and $89.1 \%$, respectively. In the group with $A D$, these values were $2.6 \%, 4.6 \%, 2.3 \%$ and $90.5 \%$, respectively. The only statistically significant difference was that of the $\mathrm{V} v$ of glial somata, which was found to be significantly higher in cases with AD ( $t$ test, $p=0.03$; Fig. 6; Extended Data Table 1-2, Table 1-3).

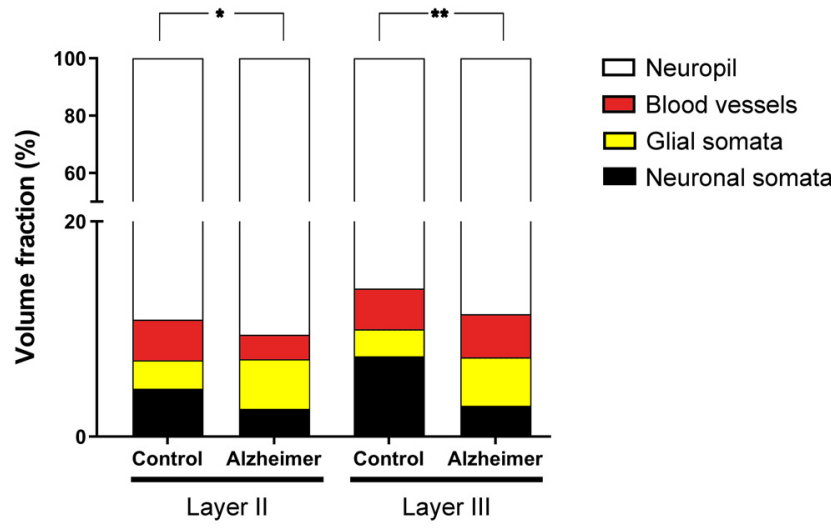

Figure 6. Graph showing the Vv occupied by neuronal and glial somata, blood vessels, and neuropil in both Layers II and III of the EC from control subjects and AD cases. Asterisks show the differences between groups. In Layer II, a significantly higher Vv of glial somata was found in AD cases ( $t$ test, $p=0.03$ ). In Layer III, a significantly lower $\mathrm{VV}$ of neuronal somata was found in AD cases ( $t$ test, $p=0.007$ ). Mean values and case values are detailed in Extended Data Table 1-2, Table 1-3.

In Layer III of the control group, the $\mathrm{V} v$ of neuronal somata, glial somata, blood vessels, and neuropil were $7.5 \%, 2.5 \%, 3.8 \%$, and $86.2 \%$. In cases with $A D$, these values were $2.9 \%, 4.6 \%, 4.0 \%$, and $88.6 \%$. Statistical comparisons showed a significantly lower $\mathrm{Vv}$ of neuronal somata ( $t$ test, $p=0.007$ ) in AD (Fig. 6; Extended Data Table 1-2, Table 1-3).

\section{Synaptic density and AS:SS ratio}

A total of 2019 synapses from AD cases (1044 synapses in Layer II and 1065 in Layer III) were identified and reconstructed in 3D, after discarding incomplete synapses or those touching the exclusion edges of the $\mathrm{CF}$, and the total volume analyzed was $9006 \mu \mathrm{m}^{3}$ in $A D$ samples (Table 2; Extended Data Table 2-1).

A significantly lower synaptic density (taking both AS and SS together) was observed in AD cases, this value was $40 \%$ lower than in control cases in both EC layers. In Layer II, we found 0.24 synapses $/ \mu \mathrm{m}^{3}$ in $A D$ cases and 0.40 synapses $/ \mu \mathrm{m}^{3}$ in control cases (MW, $p=0.03$; Fig. $7 A$ ). In Layer III, the mean synaptic density in $A D$ was 0.24 synapses $/ \mu \mathrm{m}^{3}$ compared with 0.43 synapses $/ \mu \mathrm{m}^{3}$ found in control cases (MW, $p=0.03$; Fig. 7B; Table 2; Extended Data Table 2-1).

In Layers II and III, the AS:SS ratio for the AD cases was very similar to that of the control cases (close to 92:8 in both layers and cases; Table 2; Extended Data Table 2-1), and no statistically significant differences were found between the control group and the $A D$ group in any layer $\left(\chi^{2}, p>0.05\right)$.

\section{D spatial synaptic distribution}

Analysis indicates a clear fit to a complete spatial randomness (CSR) model, since F, G, and K functions closely resemble the theoretical curve that these functions represent, both in the control and the AD cases. The CSR 
A

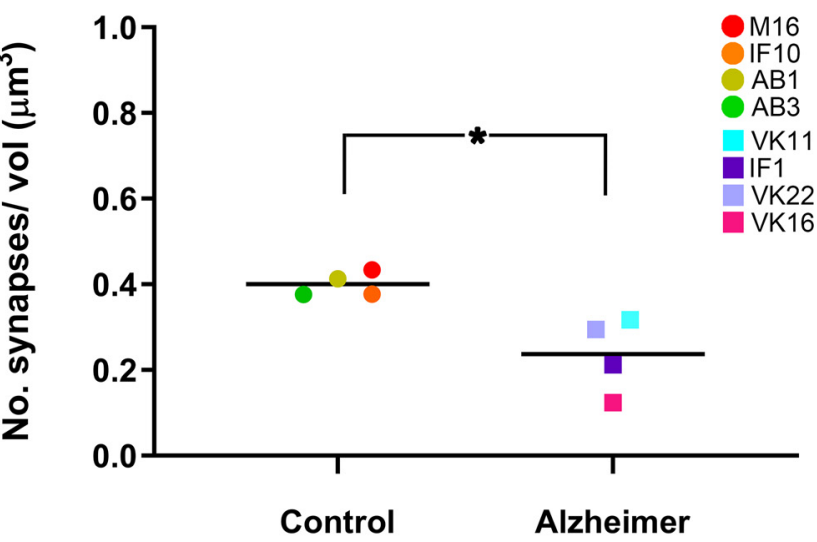

B

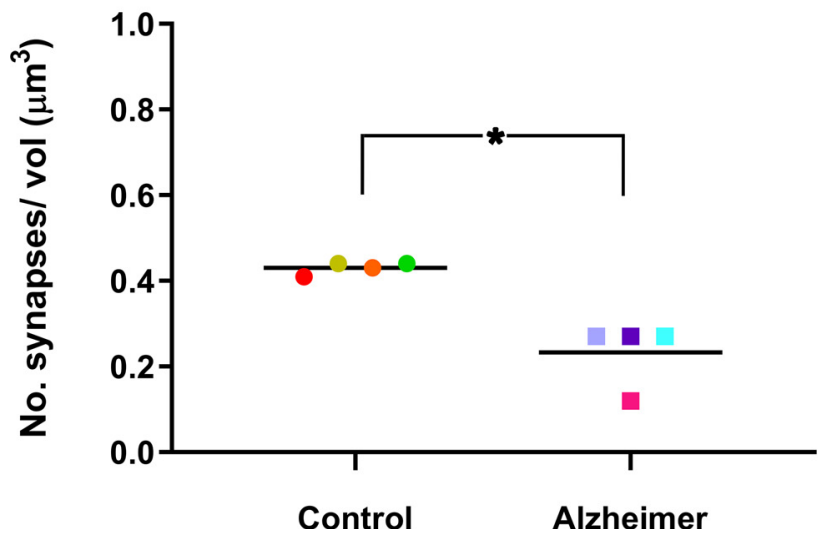

Figure 7. Graphs showing the overall mean synaptic density in Layer II $(\boldsymbol{A})$ and Layer III $(\boldsymbol{B})$ of the EC in control and AD cases. Control cases are represented by circles and AD cases are represented by squares. Each color corresponds to each case analyzed, as denoted in the upper right-hand corner. Asterisks show significant differences between groups: in both Layers II and III of the EC, the mean synaptic density was significantly lower in $A D$ cases (MW, $p=0.03$ ).

model defines a situation where a point is equally likely to occur at any location within the study volume, regardless of the locations of other points. Therefore, a CSR model is considered as a reference for a random pattern in spatial point process statistics (for review, see Merchán-Pérez et al., 2014). That is, the spatial distribution of the synapses fitted a random distribution in all subjects and layers.
Furthermore, the estimation of the distance from each synapse to its closest synapse showed that although the mean distance was greater in $A D$ cases in both Layer II $(840 \mathrm{~nm}$ in control; $938 \mathrm{~nm}$ in AD; Table 2; Extended Data Table 2-1) and Layer III (852 nm in control, $1004 \mathrm{~nm}$ in AD; Table 2; Extended Data Table 2-1), no statistically significant differences were found (MW, $p>0.05$ ).

\section{Study of the synapse characteristics \\ Synaptic size}

The AD samples did not show differences between layers in the SAS area of AS (MW, $p=0.41$; Table 3; Extended Data Table 3-1). However, we found significantly larger mean values of SAS area for AS (MW, $p=0.03$ ) from Layer II in AD cases compared with the controls. These differences were not found in SS or in Layer III (MW, $p>0.05$; Table 3; Extended Data Table 3-1).

Analysis of the frequency distributions of the area and perimeter of the AS showed significant differences in the frequency distributions of area in Layer II and in the perimeter in Layer III (KS, $p<0.0001$ ), indicating a lower proportion of small SAS area in Layer II in AD cases and higher proportions of small SAS perimeter in Layer III in AD cases (Fig. 8).

\section{Synaptic shape}

Most synapses, AS and SS, presented a macular shape in both control and AD cases in Layers II and III (>80\%), while synapses with more complex shapes (i.e., horseshoe-shaped, perforated, or fragmented) were less frequent (Fig. 9; Table 4; Extended Data Table 4-1, Table 42). Evaluation of the possible differences between the control and the AD group in Layer II showed a slightly higher proportion of horseshoe-shaped AS in AD samples $\left(\chi^{2}, p=0.04\right.$; Table 4; Extended Data Table 4-1; Fig. 9B). Analysis of Layer III revealed that fragmented $\left(\chi^{2}\right.$, $p=0.0004)$ and macular $\left(\chi^{2}, p<0.0001\right)$ AS were more frequent in the $A D$ group, whereas perforated AS were less frequent in $A D$ cases than in the control individuals $\left(\chi^{2}, p<0.0001\right.$; Table 4; Extended Data Table 4-2; Fig. $9 C)$. Analysis of the SS in Layers II and III did not reveal differences between groups $\left(\chi^{2}, p>0.001\right)$.

\section{Analysis of synaptic size and shape}

Additionally, we determined whether the shape of the synapses was related to their size. For this purpose, the area and perimeter of the SAS of AS were analyzed

Table 3: Area (mean \pm SEM, in $\mathrm{nm}^{2}$ ) and perimeter (mean $\pm \mathrm{SEM}$, in $\mathrm{nm}$ ) of the SAS in Layers II and III of the EC

\begin{tabular}{lllll}
\hline EC layer & Group & Type of synapse (no. of synapses) & Area of SAS & Perimeter of SAS \\
\hline II & Control & AS (1552) & $110,311 \pm 3229(102,865)$ & $1631 \pm 47(1575)$ \\
& & SS (139) & $65,997 \pm 6041(61,543)$ & $1404 \pm 73(1356)$ \\
& Alzheimer & AS (952) & $136,166 \pm 10,773(126,976)$ & $1819 \pm 52(1757)$ \\
III & SS (90) & AS (1746) & $59,232 \pm 6589(55,234)$ & $1239 \pm 72(1196)$ \\
& Control & SS (129) & $124,183 \pm 3265(115,801)$ & $1826 \pm 67(1763)$ \\
& Alzheimer & AS (973) & $67,445 \pm 3243(62,893)$ & $1399 \pm 75(1351)$ \\
& & SS (94) & $122,727 \pm 10,453(114,444)$ & $1720 \pm 116(1661)$ \\
& & & $57,271 \pm 14,132(56,082)$ & $1343 \pm 272(1348)$
\end{tabular}

Data on area and perimeter are corrected for shrinkage factor (data in parentheses are not corrected with the shrinkage and the fixation artifact factors). The data for individual cases are shown in Extended Data Table 3-1. 

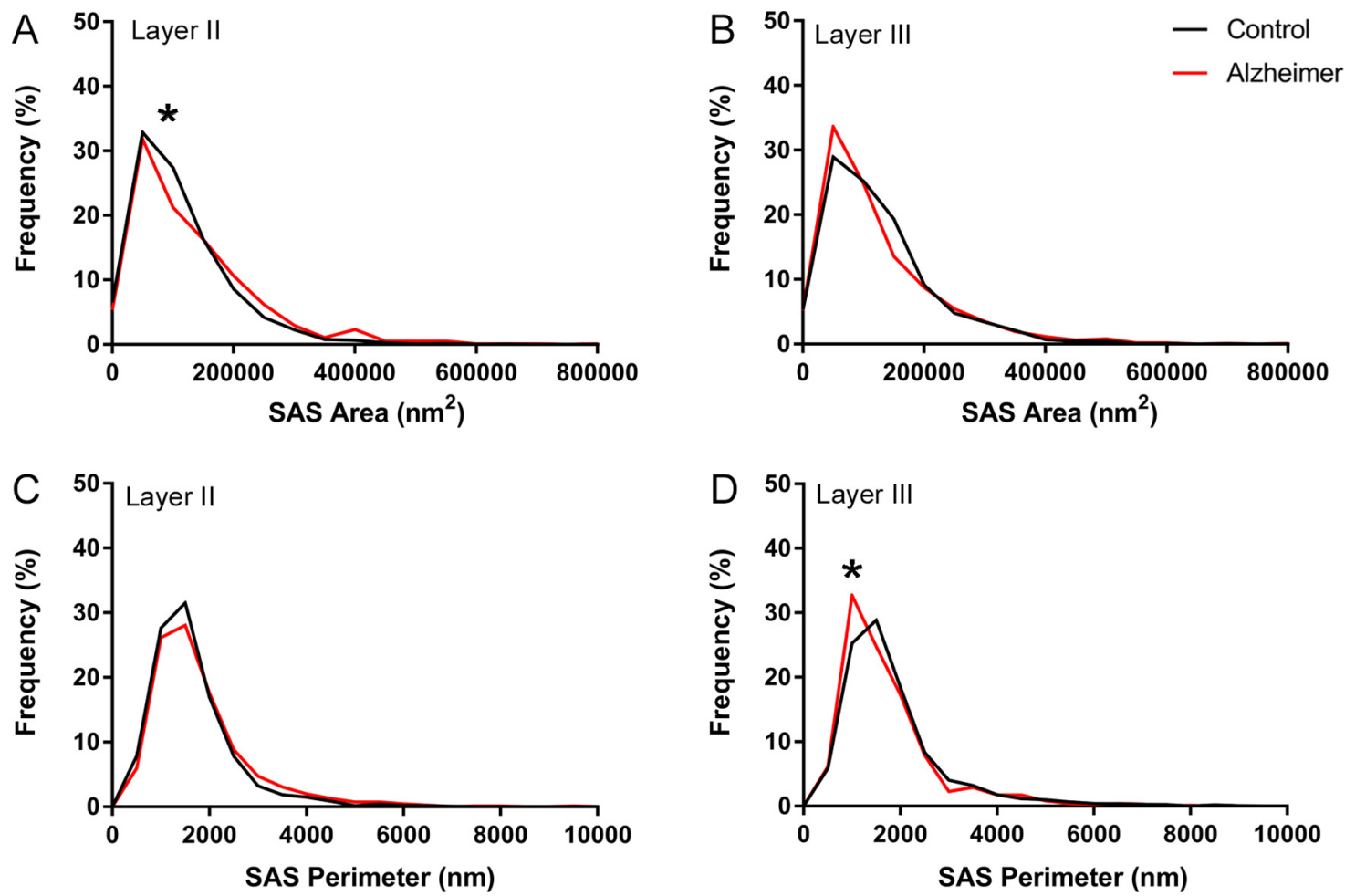

Figure 8. Graph showing the frequency distribution plots of AS SAS area $(\boldsymbol{A}, \boldsymbol{B})$ and perimeter $(\boldsymbol{C}, \boldsymbol{D})$, in Layers II and III of the EC in control subjects (black) and $A D$ cases (red). Statistical comparisons between groups showed significant differences (KS, $p<0.0001$; indicated with an asterisk) in the frequency distributions of SAS area in Layer II $(\boldsymbol{A})$, and in the frequency distribution of SAS perimeter in Layer III (D).

according to their synaptic shape (SS were not analyzed since the sample size was too small).

When we compared the synaptic size considering the synaptic shape, we found that the mean SAS area from perforated AS in Layer III was larger in $A D$ samples $\left(296,156 \pm 24,116 \mathrm{~nm}^{2}\right.$, mean \pm SEM) than in the controls $\left(228,057 \pm 6993 \mathrm{~nm}^{2}\right.$, mean \pm SEM; MW, $p=0.03$; Extended Data Tables 4-3, 4-4). No differences were found in the mean area and perimeter of the SAS from macular, horseshoe-shaped, and fragmented synapses (MW, $p>0.05$ ). Analysis of the frequency distribution of the area and perimeter showed no differences between groups (Extended Data Fig. 9-1; KS; $p>0.0001$ ).

\section{Study of the postsynaptic targets}

The postsynaptic targets of 1706 synapses were determined from the AD samples. In Layer II, we identified the postsynaptic targets of a total of 807 AS and 82 SS (Table 5; Extended Data Table 5-1), and in Layer III, the postsynaptic targets for 732 AS and 85 SS were determined (Table 5; Extended Data Table 5-2).

The most abundant type of synapse in $A D$ individuals was AS on spine heads (range 47.9-48.1\%) closely followed by AS on dendritic shafts $(41-41.8 \%)$. SS on dendritic shafts were less common (8.1-9.3\%), SS on spine heads were very uncommon $(0.7-1.1 \%)$, and AS or SS on spine necks were very infrequent indeed (<1\%; Fig. 10).
When the preference of the synaptic types (AS or SS) for a particular postsynaptic element was analyzed, we found that, in both controls and AD individuals, excitatory contacts (AS) presented a statistically significant preference for spines, whereas inhibitory contacts (SS) preferentially targeted dendritic shafts $\left(\chi^{2}, p<0.001\right)$, in both layers, in line with the findings in control EC samples from the same layers (Domínguez-Álvaro et al., 2021). Contingency tables were used to assess possible differences in the distribution of postsynaptic elements of cases with AD. No statistically significant differences were found $\left(\chi^{2}, p>0.001\right)$, although a slightly lower proportion of AS established on spine heads $\left(\chi^{2}, p=0.02\right)$ and a higher proportion of AS on dendritic shafts were observed in $A D$ samples from Layer II $\left(\chi^{2}, p=0.03\right)$.

We also analyzed whether there was a relationship between the synaptic size and the type of postsynaptic element. When we compared the synaptic size of AS associated with the postsynaptic targets between control cases and $A D$ cases, no differences were found in any layer with regard to the area and perimeter of the SAS (MW, $p>0.05$ ) or their frequency distribution (KS, $p>$ 0.001; Extended Data Fig. 10-1).

\section{Discussion}

In total, there are four main findings in the present study on the human EC: at the light microscope level, (1) a significantly lower volume fraction occupied by neuronal 
A
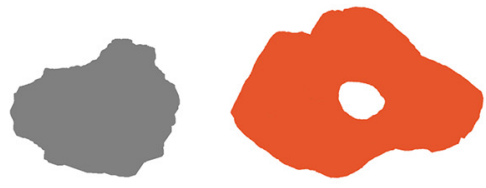

Macular

B

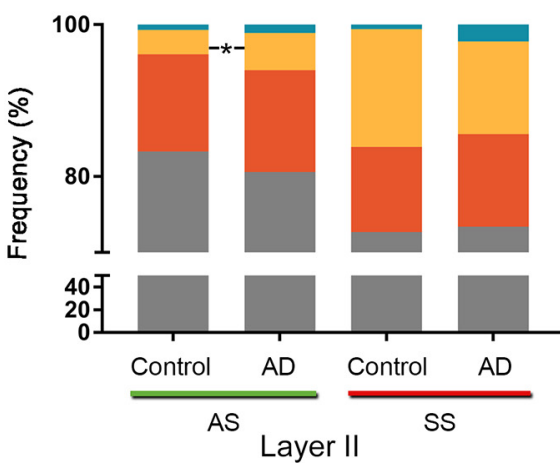

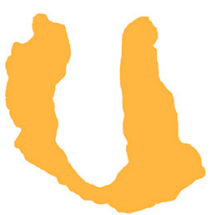

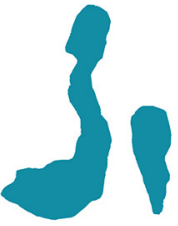

Horseshoe-shaped

Fragmented

C

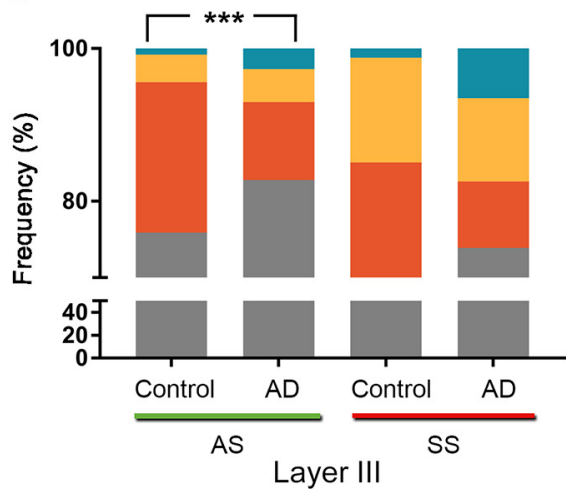

Figure 9. Proportions of the different synaptic shapes $(\boldsymbol{A})$ in Layers II $(\boldsymbol{B})$ and III $(\boldsymbol{C})$ of the EC in control subjects and AD cases. $\boldsymbol{A}$, Schematic representation of the synaptic shapes: macular synapses, with a continuous disk-shaped PSD; perforated synapses, with holes in the PSD; horseshoe-shaped, with a tortuous horseshoe-shaped perimeter with an indentation; and fragmented synapses, with two PSDs with no connections between them. Proportions of macular, perforated, horseshoe-shaped, and fragmented AS and SS are displayed for Layers II $(\boldsymbol{B})$ and III $(\boldsymbol{C})$ of the EC in control subjects and AD cases. Statistical differences (indicated with asterisks) showed that in Layer II, horseshoe-shaped AS were more frequent $\left(\chi^{2}, p=0.04\right)$ in $A D$ cases; and in Layer III of the AD group, fragmented $\left(\chi^{2}, p=0.0004\right)$ and macular $\left(\chi^{2}, p<0.0001\right)$ AS were more frequent, whereas perforated AS were less frequent in $A D$ cases than in control individuals $\left(\chi^{2}, p<0.0001\right)$.

bodies in the Layer III was found in AD cases; at the ultrastructural level, (2) a significantly lower synaptic density was found in both layers in AD cases; (3) synaptic morphology analysis revealed larger and more complex synapses in Layer II in AD cases; and (4) there was a greater proportion of small and simple synapses in Layer III in AD cases than in control individuals.

\section{Cortical thickness and volume fraction analysis}

At the light microscopic level, atrophy of the EC and adjacent cortical regions is clearly visible to the naked eye (Fig. 1). However, the difference in the mean cortical thickness was no evident (Extended Data Table 1-1). Macroscopic atrophy of the EC has been described in $A D$ cases (Van Hoesen et al., 1991). This cortical atrophy and, more specifically, the neuronal loss (see below) observed in $A D$ cases have been related to the presence of NFTs and $\mathrm{A} \beta$ plaques, with reports indicating an inverse relationship between the number of neurons and the degree of neuropathology (Van Hoesen et al., 1991; Gómez-Isla et al., 1996).

In AD brain samples, we found a significant lower neuronal volume fraction in the Layer III. This is in line with the reported neuronal loss from AD cases in the EC (Van Hoesen and Hyman, 1990; Gómez-Isla et al., 1996; Šimić et al., 2017). Since the neurons of EC Layer III constitute the projection elements of the perforant pathway to the hippocampal CA1 (together with neurons from Layer II), this neuronal loss may contribute to the memory

Table 4: Proportion of the different synaptic shapes in Layers II and III of the EC

\begin{tabular}{|c|c|c|c|c|c|c|c|}
\hline $\begin{array}{l}\mathrm{EC} \\
\text { layer }\end{array}$ & Group & $\begin{array}{l}\text { Type of } \\
\text { synapse }\end{array}$ & $\begin{array}{l}\text { Macular } \\
\text { synapses }\end{array}$ & $\begin{array}{l}\text { Perforated } \\
\text { synapses }\end{array}$ & $\begin{array}{l}\text { Horseshoe } \\
\text { synapses }\end{array}$ & $\begin{array}{l}\text { Fragmented } \\
\text { synapses }\end{array}$ & $\begin{array}{l}\text { Total } \\
\text { synapses }\end{array}$ \\
\hline \multirow{2}{*}{ II } & & SS & $73.0 \%(100)$ & $11.0 \%(15)$ & $15.3 \%(21)$ & $0.7 \%(1)$ & $100 \%(137)$ \\
\hline & & SS & $73.4 \%(66)$ & $12.2 \%(11)$ & $12.2 \%(11)$ & $2.2 \%(2)$ & $100 \%(90)$ \\
\hline \multirow[t]{2}{*}{ III } & Control & AS & $75.9 \%(1326)$ & $19.9 \%(347)$ & $3.3 \%(58)$ & $0.9 \%(15)$ & $100 \%(1746)$ \\
\hline & & SS & $65.9 \%(85)$ & $15.5 \%(20)$ & $17.0 \%(22)$ & $1.6 \%(2)$ & $100 \%(129)$ \\
\hline
\end{tabular}

Data are given as percentages with the absolute number of synapses studied in parentheses. Data for individual cases are shown in Extended Data Table 4-1, Table 4-2. 
Table 5: Distribution of AS and SS on spines and dendritic shafts in Layers II and III of the EC

\begin{tabular}{|c|c|c|c|c|c|c|c|c|}
\hline Layer & Group & $\begin{array}{l}\text { Type of } \\
\text { synapse }\end{array}$ & $\begin{array}{l}\text { On spine } \\
\text { heads }\end{array}$ & $\begin{array}{l}\text { On nf. } \\
\text { spines }\end{array}$ & $\begin{array}{l}\text { On spine } \\
\text { necks }\end{array}$ & $\begin{array}{l}\text { On aspiny } \\
\text { dendritic } \\
\text { shaft }\end{array}$ & $\begin{array}{l}\text { On spiny } \\
\text { dendritic } \\
\text { shaft }\end{array}$ & Total \\
\hline \multirow[t]{3}{*}{$\overline{\text { II }}$} & Control & AS & $39.68 \%(567)$ & $18.89 \%(270)$ & $0.56 \%(8)$ & $19.87 \%$ (284) & $20.99 \%(300)$ & $100 \%(1429)$ \\
\hline & Alzheimer & AS & $35.19 \%(284)$ & $17.84 \%(144)$ & $0.87 \%(7)$ & $25.15 \%(203)$ & $20.94 \%$ (169) & $100 \%(807)$ \\
\hline & & SS & $10.98 \%(9)$ & $1.22 \%(1)$ & $0 \%(0)$ & $48.78 \%(40)$ & $39.02 \%(32)$ & $100 \%(82)$ \\
\hline \multirow[t]{2}{*}{ III } & Control & AS & $39.68 \%(642)$ & $15.33 \%(248)$ & $0.74 \%(12)$ & $17.49 \%(283)$ & $26.79 \%(433)$ & $100 \%(1618)$ \\
\hline & & SS & $5.88 \%(5)$ & $1.18 \%(1)$ & $3.53 \%(3)$ & $40 \%(34)$ & $49.41 \%(42)$ & $100 \%(85)$ \\
\hline
\end{tabular}

Synapses on spines have been subdivided into those that are established on spine heads and those that are established on spine necks. Moreover, we differentiated between aspiny and spiny dendritic shafts. Data are expressed as percentages with the absolute number of synapses studied given in parentheses. Data for each individual case are shown in Extended Data Table 5-1, Table 5-2. nf.: non-fully reconstructed.

impairment described in AD cases (Hyman et al., 1986; Van Hoesen et al., 1991; von Gunten et al., 2006; Andrade-Moraes et al., 2013). Since our present neuronal volume fraction estimations are based on semithin sections, and different types of neurons have not been identified, we cannot rule out a selective neuronal loss that may affect either to excitatory neurons or to GABAergic interneurons or to both cell types.

The significantly higher volume fraction occupied by glial somata found in Layer II is in line with previous reports showing a larger density of glial cells in human EC from AD cases (Muramori et al., 1998). However, no significant differences were found in Layer III, suggesting a layer-selective change. The effects of gliosis during AD development are still under debate. It has been proposed that gliosis mediates an inflammatory response to prevent the progression of the disease but that this response may in fact contribute to neurodegeneration (for review, see Fakhoury, 2018).

\section{Synaptic changes in AD}

Synaptic density, proportions, and spatial distribution

Our main finding was a significantly lower synaptic density (around 40\% lower) in the neuropil of both Layers II and III of AD individuals compared with controls.
In brain tissue samples from individuals with $A D$, a lower density of synapses has been described compared with controls in numerous cortical regions, including the DG and certain frontal, parietal, temporal and cingulate cortical areas (Scheff et al., 1990, 1996; Scheff and Price, 1993, 1998, 2003, 2006). A decrease in the total number of synapses, accompanied by brain atrophy, has been also reported in $\mathrm{CA} 1$, the DG and some cingulate and temporal cortices (Scheff et al., 2006, 2007, 2011, 2015; Montero-Crespo et al., 2021). However, other studies have not found differences in synaptic density in layers III and $\mathrm{V}$ of EC samples from AD cases (Scheff et al., 1993). These discrepancies may be because of differences in the methodologies used. Furthermore, numerous studies have estimated the synaptic density using indirect methods, either using light microscopy to count immunoreactive puncta for synaptic markers (Masliah et al., 1990; Honer et al., 1992), or employing conventional transmission EM to examine single or few serial ultrathin sections (Scheff et al., 1993). Quantification of synaptic density in single ultrathin sections using transmission EM to infer 3D characteristics of synaptic junctions observed in two dimensions may lead to inaccurate synaptic density estimations (see Merchán-Pérez et al., 2009). Thus, the present results revealing a lower number of synapses per volume of neuropil, along with the atrophy of the EC compared with controls, suggest a dramatic decrease in the
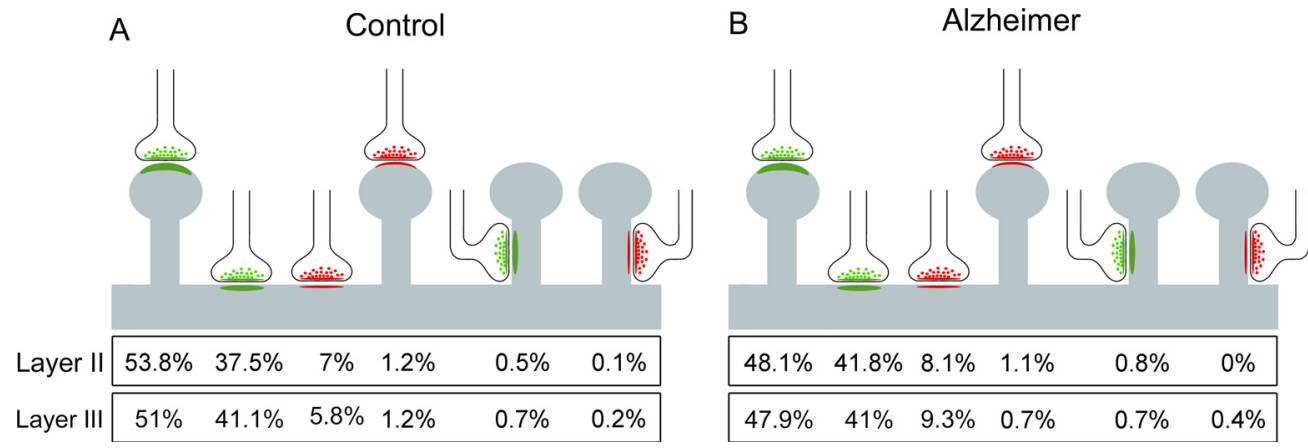

Figure 10. Postsynaptic target distribution in Layers II and III of the EC. Schematic representation of the distribution of AS and SS on different postsynaptic targets from control cases $(\boldsymbol{A})$ and AD cases $(\boldsymbol{B})$. In both Layers II and III, the AS preferentially targeted spine heads, while the SS preferentially targeted dendritic shafts. Percentages of postsynaptic targets are indicated, showing, from left to right, the most frequent type (AS on spine heads) to the least frequent type (SS on spine necks). Synapses on spines have been subclassified into those that are established on the spine head and those established on the neck. 
absolute number of synapses in $A D$ cases in these $E C$ layers.

Synaptic loss has been widely reported as the characteristic that best correlates with cognitive deficit in $A D$ cases (for review, see Colom-Cadena et al., 2020). Synaptic loss occurs in the early stages of the disease, affecting firstly the subcortical regions and the EC, and progressing to other cortical regions (Braak and Braak, 1991; Braak and Del Tredici, 2012). Tau protein and $\beta$-amyloid peptide in pathologic conditions could have toxic effects on synapses leading to synaptic loss and/or dysfunction in AD (Zhou et al., 2017; for review, see Henstridge et al., 2016; Rajmohan and Reddy, 2017). This synaptic loss and impairment may cause dysfunction in the cortical circuits contributing to the decline in cognition (for review, see Colom-Cadena et al., 2020).

Previous studies have shown that the percentage of AS and SS varies, $80-95 \%$ and $20-5 \%$, respectively, in all the cortical layers, cortical areas and species examined so far by transmission EM (Beaulieu and Colonnier, 1985; Megías et al., 2001; Bourne and Harris, 2011; DeFelipe, 2011, 2015). Estimations with samples from human brain using the same method have shown that the AS:SS ratio in Layer III of the temporal area 21 is 93:7 (Cano-Astorga et al., 2021), 96:4 in the transentorhinal cortex (Domínguez-Álvaro et al., 2018), and 95:5 in the human CA1 hippocampal field (except in the stratum lacunosum moleculare, in which this ratio was 89:11; MonteroCrespo et al., 2020). Thus, it would appear that the present AS:SS ratio data provide among the highest and lowest proportions previously observed in different brain regions, for AS and SS, respectively. From a functional point of view, the proportion of excitatory and inhibitory synapses is critical, since higher or lower proportions are linked to differences in the excitatory/inhibitory balance of the cortical circuits (for review, see Froemke, 2015; Zhou and Yu, 2018; Sohal and Rubenstein, 2019). Interestingly, no changes in the proportions of AS and SS were observed; therefore, the lower synaptic density in AD cases might affect AS and SS equally, which is in line with our previous results in the transentorhinal cortex (DomínguezÁlvaro et al., 2018). Since most of the synapses are excitatory synapses ( $\sim 92 \% \mathrm{AS})$, the synaptic decrease may result in a massive loss of AS in particular. As the present results did not reveal differences in the AS:SS ratio between control and $A D$ cases in the neuropil in any layer, it could be interpreted that the proportion of AS and SS on the dendritic arbor of the different types of neurons may be similar. However, it has been shown that there are differences in the number of GABAergic and glutamatergic synaptic inputs in different neuronal types in other cortical regions of a variety of species (DeFelipe and Fariñas, 1992; Freund and Buzsáki, 1996; DeFelipe, 1997; Somogyi et al., 1998; Schubert et al., 2007; Markram et al., 2015; Tremblay et al., 2016; Hsu et al., 2017). Thus, it would be necessary to examine the synaptic inputs on each specific neuronal type to determine actual differences in the AS:SS ratio in particular cell types, although the final general AS:SS ratio does not vary in the neuropil.

Furthermore, it has been reported that GABA inhibitory interneurons in the brains of $A D$ cases are abnormally reduced, along with the inhibitory neurotransmitters (for review, see Xu et al., 2020). Other studies have shown that somatostatin neurons, calretinin neurons, and parvalbumin neurons are decreased in several brain regions including the EC of AD cases (Solodkin and Van Hoesen, 1996; Mikkonen et al., 1999). Therefore, further studies should be performed to examine the GABAergic innervation of neurons in the EC.

Finally, the present results indicate that the distribution of synapses in the neuropil of both control and AD samples is nearly random, only constrained by the fact that synapses cannot physically overlap in space and so their geometric centers or centroids cannot be too close to their neighbors. As we have previously shown in human brain samples from hippocampal CA1, EC, transentorhinal cortex and temporal cortex, there seems to be no limitation to the position of any synapse except the space already occupied by other synapses (Domínguez-Álvaro et al., 2018, 2021; Montero-Crespo et al., 2020; CanoAstorga et al., 2021). Previous studies in the plaque-free neuropil of $A D$ cases also showed a random distribution pattern of the synapses in other brain regions (BlazquezLlorca et al., 2013; Domínguez-Álvaro et al., 2018; Montero-Crespo et al., 2021). It has been reported that during cortical development, synapses are randomly added and/or withdrawn from the population, as described by the synaptic turnover (Rakic et al., 1994). It seems that the only constraint for a synapse to form is that this particular spot is not already occupied by a preexisting synapse (for review, see Merchán-Pérez et al., 2014). Interestingly, in the AD cases, the loss of synapses per unit volume does not affect their 3D distribution.

\section{Shape and size of the synapses}

There are very few studies on human brain at the ultrastructural level that provide 3D data on the morphologic characteristics of the synaptic junctions to compare with our findings. In the examined EC layers, excitatory AS were larger than inhibitory SS, as previously shown in human transentorhinal and temporal cortex (DomínguezÁlvaro et al., 2018; Cano-Astorga et al., 2021) and in several strata from the hippocampal CA1 (Montero-Crespo et al., 2020). Furthermore, no differences between layers were found regarding the SAS area of $A S$ in $A D$ cases $\left(136,166 \mathrm{~nm}^{2}\right.$ in Layer II and $122,727 \mathrm{~nm}^{2}$ in Layer III), which was in contrast with previous results in control EC showing larger synapses in Layer III (Domínguez-Álvaro et al., 2021). However, the synaptic size in AD samples differs from that of control individuals, with larger and more complex synapses in Layer II and a greater proportion of smaller synapses in Layer III.

Several EM studies conducted with samples of human brain tissue have reported an increase in the size of synaptic apposition, and this has been suggested as a possible compensatory mechanism for the synaptic loss (for review, see Scheff and Price, 2006). Models studying the relationship between the size of synaptic junctions and the probability of neurotransmitter release suggest that larger synapses (with a greater number of postsynaptic receptors, mainly AMPA receptors) may produce more powerful, homogeneous and numerous 
synaptic responses, while smaller synapses (with fewer receptors, mainly NMDA) may produce weaker and more variable responses (Kharazia and Weinberg, 1999; Montes et al., 2015). Thus, alterations in the normal size of the synaptic junction may change the proportion of postsynaptic receptors, thereby modifying the synaptic response.

Regarding synaptic shape, we found a higher proportion of horseshoe synapses in Layer II from AD cases. In Layer III from these AD individuals, higher proportion of AS with macular and fragmented shape were found along with a lower proportion of perforated AS synapses. Several studies have linked an increase in synaptic complexity to an increase in the efficiency of synaptic transmission, via the insertion of new receptors in the postsynaptic membrane (Lüscher et al., 2000). Perforated synapses have higher immunoreactivity for glutamate receptors than non-perforated synapses (Ganeshina et al., $2004 a, b)$. The larger proportion of macular AS in Layer III (around $83 \%$ in $\mathrm{AD}$ cases, vs $76 \%$ in control cases) and the corresponding lower number of perforated synapses (about half the number found in control cases) supports the evidence for a larger number of simple synapses in this layer in AD cases, which is in turn supported by the finding of a higher proportion of smaller synapses described above. However, the higher proportion of fragmented AS in Layer III from AD cases may indicate the above-mentioned compensatory response to the synaptic loss. Conversely, small macular synapses from Layer III may be more susceptible to damage resulting in a reduction in their numbers. That is, synaptic morphology may be selectively altered by layer, with a non-homogenous pattern of changes, which would differentially affect the circuits in which these layers are involved.

Therefore, synaptic changes in Layers II and III might be part of a remodeling process of the remaining synapses, which overcomes the overall synaptic loss. If the remodeling process involves an increase in the proportion of synapses with more complex morphology, it could be hypothesized that the morphologic changes in $A D$ would trigger compensatory mechanisms during the progression of the disease. However, this type of compensatory mechanism may fail or be deficient, leading to a decline in the synaptic functionality accompanying the progression of the disease.

\section{Postsynaptic targets}

Despite the lower synaptic density in AD cases, no changes in the distribution and types of postsynaptic targets were found. In the AD samples, the most frequent combinations were axospinous AS (on dendritic spine heads) and axodendritic AS (established on dendritic shafts). In addition, the majority of the synapses that a pyramidal cell receives are on spines and they represent the vast majority of AS synapses (DeFelipe and Fariñas, 1992). Using the same FIB/SEM technology, in Layer II of the human transentorhinal cortex, it was reported that $60 \%$ of all AS were established on spines that were fully reconstructed (Domínguez-Álvaro et al., 2019). However, when we re-analyzed the stacks of images of the TEC to include the non-fully reconstructed spines, the AS percentage established on dendritic spine heads was $75 \%$, which is similar to the case of the AS found in Layer III of the temporal cortex (75\%) using the same analysis method (Cano-Astorga et al., 2021). This percentage was much higher in the CA1 hippocampal field, as high as $94 \%$ in the superficial part of the CA1 stratum pyramidale (Montero-Crespo et al., 2020). In both Layers II and III of the EC, the percentage of AS on spines was $57 \%$, and $43 \%$ in the case of AS on dendritic shafts. This is a higher proportion of AS on shafts than in the other human cortical regions previously analyzed using the same techniques.

In addition, the comparison between control and $A D$ samples did not show a clear reduction in the proportion of AS on spines. Only a slightly lower proportion of AS established on spines was found in Layer II from AD samples. Therefore, the lower synaptic density does not seem to be specifically related to a loss of spines, but rather a general loss of synapses might occur regardless of their postsynaptic targets. It may be that the axospinous synapses in EC from $A D$ cases are functionally altered although no changes were found in their proportions.

\section{Implications of the synaptic changes}

EC provides excitatory inputs to the hippocampus from Layer II neurons targeting the DG (trisynaptic pathway) and from Layer III neurons monosynaptically targeting CA1 (for review, see Insausti and Amaral, 2012; Marks et al., 2020). Neuronal projections from both layers give rise to the perforant pathway, which has generally been reported to be weakened in AD (Hyman et al., 1986).

Moreover, in Layer II there are two subpopulations of excitatory neurons: spiny stellate neurons (which project to DG, CA3 and CA2) and modified pyramidal neurons (which project to stratum lacunosum in CA1). Projections from these neurons, constituting the trisynaptic pathway, seem to be related to contextual memories (for review, see Marks et al., 2020). It has been proposed that this pathway is more susceptible to premature degeneration than the monosynaptic pathway (Van Hoesen et al., 2006; Llorens-Martín et al., 2014).

In addition, pyramidal neurons from Layer III directly project to pyramidal neuron dendrites located in the stratum moleculare of CA1, and it has been proposed that these monosynaptic excitatory inputs drive temporal association learning (for review, see Marks et al., 2020).

The present study shows a significant lower number of synapses per volume, as well as morphologic synaptic alterations in the EC from AD cases in Layers II and III. Since the AD cases examined in the present study correspond to advanced stages of the disease, we do not know when the synaptic alterations occurred. Considering that there is neuronal loss, the lower number of synapses may be explained, at least in part, by the loss of local axons of these neurons, at least at the earlier stages of the disease. However, the lower number of synapses may also be explained by a loss of projection neurons that die in distant cortical regions that are also affected by the disease. 


\section{Interindividual variability}

The present data cannot be generalized to the whole population of patients with $A D$, which shows clear variability between individuals (Fig. 7; Table 2). Therefore, our study can be considered as a further step to tackle the issue of the synaptic alterations in $A D$ cases, but it would be necessary to validate our results, both in a larger number of individuals and in additional brain regions (which we have already done in the hippocampal CA1 field and in the transentorhinal cortex). Technical effects could be ruled out given that the postmortem delays were all similar and the procedures used were the same. Moreover, all of the $A D$ cases examined were women aged between 80 and 86 years old; therefore, variability because of sex and aging could also be ruled out.

Considering the above-mentioned toxic effect of tau protein and $\beta$-amyloid peptide on the synapses, a relationship between the degree of disease progression and the synaptic loss might be expected: the greater the degree of the pathology, the greater the synaptic loss. Subject VK16, who presented a high score for neuropathology and disease progression (Braak/CERAD: VI/C), also had the lowest synaptic density in both layers $(0.12$ synapse/ $\mu \mathrm{m}^{3}$; Fig. 7; Extended Data Table 2-1). However, subject VK22, who also had a high score for neuropathology and disease progression (V/C), had a synaptic density of more than double $\left(0.29\right.$ and 0.27 synapse $/ \mu \mathrm{m}^{3}$, for Layers II and III, respectively) and both cases, VK16 and VK22, suffered dementia. However, subject IF1 did not present evidence of dementia despite the fact that this subject had a high level of pathology (IV/B) and low synaptic density, which was similar to VK22 (0.21 and 0.27 synapse $/ \mu^{3}{ }^{3}$, for Layers II and III, respectively). However, the cortical thickness of case IF1 was $2.3 \mathrm{~mm}$, whereas in VK16 and VK22, the cortical layers were thinner (1.19 and $1.51 \mathrm{~mm}$, respectively; Extended Data Table 1-1). As pointed out by Ferrer (2012), it should be kept in mind that $A D$, at least and limited to the entorhinal and transentorhinal cortices (Stages I-II), affects $\sim 80 \%$ of individuals over 65 years, but dementia only occurs in a small percentage of individuals in this age bracket (the prevalence of dementia in $A D$ increases to $25 \%$ in 80 -year-old individuals). Thus, it is possible that this particular case (IF1) may represent a predementia stage of $A D$ (prodromal $A D$ ).

Control cases were on average younger ( 51 years old) than $A D$ cases (85years old), and it remains uncertain whether some of the synaptic alterations observed in the present work were because of normal aging or because of the pathology per se. Although no sex-based differences have been reported with aging in the human cerebral cortex (Scheff et al., 2001), previous studies on synaptic density in the human temporal cortex have shown differences depending on the sex (Alonso-Nanclares et al., 2008). Nevertheless, no similar EM studies have been performed in the EC in individuals with different ages and sexes. Thus, the lower synaptic density found in $A D$ cases might also be the results of sex or age effects. In aged rhesus monkey, a lower number of synapses has been reported in prefrontal cortex related to a cognitive decline; however, studies in rats and monkeys have shown no evidence of synaptic loss with age in mesial temporal lobe structures (for review, see Morrison and Baxter, 2012). Further studies using human brain tissue from older control cases would be necessary to determine whether or not these differences in the synaptic density are because of these factors, as opposed to the AD per se. Nevertheless, it is important to point out that only the neuropil without plaques was examined in the present study, whereas it is well established that the neuropil which is adjacent to, and is surrounding, the plaques shows synaptic alterations (BlazquezLlorca et al., 2013). Thus, it is possible that the neuropil lacking plaques in AD cases displays "normal" characteristics, and differences observed in our study comparing to the control group might be because of factors related to sex and age.

In the present study, we found, in addition to synaptic loss, changes in the morphology of the synapses in $A D$ compared with control cases. These structural changes may contribute to the anatomic basis for the impairment of cognitive functions in AD. Despite the large number of synapses analyzed (5000 synapses), and the proven robustness of the methodology used in this study, it should be kept in mind that (1) the data comes from the analysis of four control cases and four AD cases and (2) we cannot rule out sex-related and age-related factors that may influence the differences observed between the two groups. In order to address these remaining issues, it would be necessary to perform further studies using FIB/ SEM or other similar techniques to reconstruct thousands of synapses in aged control cases of both sexes.

\section{References}

Alonso-Nanclares L, Gonzalez-Soriano J, Rodriguez JR, DeFelipe J (2008) Gender differences in human cortical synaptic density. Proc Natl Acad Sci USA 105:14615-14619.

Alzheimer's Association (2020) Alzheimer's disease facts and figures. Alzheimer Dement 16:391.

Andrade-Moraes $\mathrm{CH}$, Oliveira-Pinto AV, Castro-Fonseca E, da Silva CG, Guimarães DM, Szczupak D, Parente-Bruno DR, Carvalho LRB, Polichiso L, Gomes BV, Oliveira LM, Rodriguez RD, Leite REP, Ferretti-Rebustini REL, Jacob-Filho W, Pasqualucci CA, Grinberg LT, Lent R (2013) Cell number changes in Alzheimer's disease relate to dementia, not to plaques and tangles. Brain 136:3738-3752.

Baddeley A, Rubak E, Turner R (2015) Spatial point patterns: methodology and applications with R. Boca Raton: Chapman and Hall/ CRC.

Beaulieu C, Colonnier M (1985) A laminar analysis of the number of round-asymmetrical and flat-symmetrical synapses on spines, dendritic trunks, and cell bodies in area 17 of the cat. J Comp Neurol 231:180-189.

Blazquez-Llorca L, Merchán-Pérez Á, Rodríguez JR, Gascón J, DeFelipe J (2013) FIB/SEM technology and AD: three-dimensional analysis of human cortical synapses. J Alzheimers Dis 34:9951013.

Blazquez-Llorca L, Woodruff A, Inan M, Anderson SA, Yuste R, DeFelipe J, Merchan-Perez A (2015) Spatial distribution of neurons innervated by chandelier cells. Brain Struct Funct 220:2817-2834.

Bourne JN, Harris KM (2011) Coordination of size and number of excitatory and inhibitory synapses results in a balanced structural plasticity along mature hippocampal CA1 dendrites during LTP. Hippocampus 21:354-373. 
Braak H, Braak E (1991) Neuropathological stageing of Alzheimer-related changes. Acta Neuropathol 82:239-259.

Braak H, Braak E (1992) The human entorhinal cortex: normal morphology and lamina-specific pathology in various diseases. Neurosci Res 15:6-31.

Braak H, Del Tredici K (2012) Where, when, and in what form does sporadic Alzheimer's disease begin? Curr Opin Neurol 25:708714.

Braak H, Del Tredici K (2020) From the entorhinal region via the prosubiculum to the dentate fascia: Alzheimer disease-related neurofibrillary changes in the temporal allocortex. J Neuropathol Exp Neurol 79:163-175.

Cano-Astorga N, DeFelipe J, Alonso-Nanclares L (2021) Three-dimensional synaptic organization of layer III of the human temporal neocortex. Cereb Cortex. Advance online publication. Retrieved May 17, 2021. doi: 10.1093/cercor/bhab120.

Chen Y, Fu AKY, Ip NY (2019) Synaptic dysfunction in Alzheimer's disease: mechanisms and therapeutic strategies. Pharmacol Ther 195:186-198.

Coleman P, Federoff H, Kurlan R (2004) A focus on the synapse for neuroprotection in Alzheimer disease and other dementias. Neurology 63:1155-1162.

Colom-Cadena M, Spires-Jones T, Zetterberg H, Blennow $\mathrm{K}$, Caggiano A, DeKosky ST, Fillit H, Harrison JE, Schneider LS, Scheltens P, de Haan W, Grundman M, van Dyck CH, Izzo NJ, Catalano SM; Synaptic Health Endpoints Working Group (2020) The clinical promise of biomarkers of synapse damage or loss in Alzheimer's disease. Alzheimers Res Ther 12:21.

DeFelipe J (1997) Types of neurons, synaptic connections and chemical characteristics of cells immunoreactive for calbindinD28K, parvalbumin and calretinin in the neocortex. J Chem Neuroanat 14:1-19.

DeFelipe $J$ (2011) The evolution of the brain, the human nature of cortical circuits and intellectual creativity. Front Neuroanat 5:29.

DeFelipe J (2015) The anatomical problem posed by brain complexity and size: a potential solution. Front Neuroanat 9:104.

DeFelipe J, Fairén A (1993) A simple and reliable method for correlative light and electron microscopic studies. J Histochem Cytochem 41:769-772.

DeFelipe J, Fariñas I (1992) The pyramidal neuron of the cerebral cortex: morphological and chemical characteristics of the synaptic input. Prog Neurobiol 39:563-607.

del Río MR, DeFelipe J (1995) A light and electron microscopic study of calbindin D-28k immunoreactive double bouquet cells in the human temporal cortex. Brain Res 690:133-140.

Dickson DW (1997) The pathogenesis of senile plaques. J Neuropathol Exp Neurol 56:321-339.

Dickson DW, Crystal HA, Bevona C, Honer W, Vincent I, Davies P (1995) Correlations of synaptic and pathological markers with cognition of the elderly. Neurobiol Aging 16:285-304.

Domínguez-Álvaro M, Montero-Crespo M, Blazquez-Llorca L, Insausti R, DeFelipe J, Alonso-Nanclares L (2018) Three-dimensional analysis of synapses in the transentorhinal cortex of Alzheimer's disease patients. Acta Neuropathol Commun 6:20.

Domínguez-Álvaro M, Montero-Crespo M, Blazquez-Llorca L, DeFelipe J, Alonso-Nanclares L (2019) 3D electron microscopy study of synaptic organization of the normal human transentorhinal cortex and its possible alterations in Alzheimer's disease. eNeuro 6:ENEURO.0140-19.2019-17.

Domínguez-Álvaro M, Montero-Crespo M, Blazquez-Llorca L, DeFelipe J, Alonso-Nanclares L (2021) 3D ultrastructural study of synapses in the human entorhinal cortex. Cereb Cortex 31:410425.

Fakhoury M (2018) Microglia and astrocytes in Alzheimer's disease: implications for therapy. Curr Neuropharmacol 16:508-518.

Ferrer I (2012) Defining Alzheimer as a common age-related neurodegenerative process not inevitably leading to dementia. Prog Neurobiol 97:38-51.
Forner S, Baglietto-Vargas D, Martini AC, Trujillo-Estrada L, LaFerla FM (2017) Synaptic impairment in Alzheimer's disease: a dysregulated symphony. Trends Neurosci 40:347-357.

Freund TF, Buzsáki G (1996) Interneurons of the hippocampus. Hippocampus 6:347-470.

Froemke RC (2015) Plasticity of cortical excitatory-inhibitory balance. Annu Rev Neurosci 38:195-219.

Ganeshina O, Berry RW, Petralia RS, Nicholson DA, Geinisman Y (2004a) Differences in the expression of AMPA and NMDA receptors between axospinous perforated and nonperforated synapses are related to the configuration and size of postsynaptic densities. J Comp Neurol 468:86-95.

Ganeshina O, Berry RW, Petralia RS, Nicholson DA, Geinisman Y (2004b) Synapses with a segmented, completely partitioned postsynaptic density express more AMPA receptors than other axospinous synaptic junctions. Neurosci 125:615-623.

Gómez-Isla T, Price JL, McKeel DW, Morris JC, Growdon JH, Hyman BT (1996) Profound loss of layer II entorhinal cortex neurons occurs in very mild Alzheimer's disease. J Neurosci 16:4491-4500.

Gray EG (1959) Axo-somatic and axo-dendritic synapses of the cerebral cortex: an electron microscope study. J Anat 93:420433.

Gundersen HJ, Bendtsen TF, Korbo L, Marcussen N, Møller A, Nielsen K, Nyengaard JR, Pakkenberg B, Sørensen FB, Vesterby A (1988) Some new, simple and efficient stereological methods and their use in pathological research and diagnosis. APMIS 96:379394.

Henstridge CM, Pickett E, Spires-Jones TL (2016) Synaptic pathology: a shared mechanism in neurological disease. Ageing Res Rev 28:72-84.

Honer WG, Dickson DW, Gleeson J, Davies P (1992) Regional synaptic pathology in AD. Neurobiol Aging 13:375-382.

Hsu A, Luebke JI, Medalla M (2017) Comparative ultrastructural features of excitatory synapses in the visual and frontal cortices of the adult mouse and monkey. J Comp Neurol 525:21752191.

Hyman BT, Van Hoesen GW, Kromer LJ, Damasio AR (1986) Perforant pathway changes and the memory impairment of Alzheimer's disease. Ann Neurol 20:472-481.

Insausti R, Amaral DG (2012) Hippocampal formation. In: The human nervous system (Mai JK, Paxinos G, eds), pp 896-942. Cambridge: Academic Press.

Insausti R, Muñoz-López M, Insausti AM, Artacho-Pérula $E$ (2017) The human periallocortex: layer pattern in presubiculum, parasubiculum and entorhinal cortex. A review. Front Neuroanat 11:84

Kharazia VN, Weinberg RJ (1999) Immunogold localization of AMPA and NMDA receptors in somatic sensory cortex of albino rat. $J$ Comp Neurol 412:292-302.

Kobro-Flatmoen A, Witter MP (2019) Neuronal chemo-architecture of the entorhinal cortex: a comparative review. Eur J Neurosci 50:3627-3662.

Kondo H, Lavenex P, Amaral DG (2009) Intrinsic connections of the macaque monkey hippocampal formation: II. CA3 connections. J Comp Neurol 515:349-377.

Lavenex P, Amaral DG (2000) Hippocampal-neocortical interaction: a hierarchy of associativity. Hippocampus 10:420-430.

Llorens-Martín M, Blazquez-Llorca L, Benavides-Piccione R, Rabano A, Hernandez F, Avila J, DeFelipe J (2014) Selective alterations of neurons and circuits related to early memory loss in AD. Front Neuroanat 8:38.

Lüscher C, Nicoll RA, Malenka RC, Muller D (2000) Synaptic plasticity and dynamic modulation of the postsynaptic. Nat Neurosci 3:545-550.

Marks WD, Yamamoto N, Kitamura T (2020) Complementary roles of differential medial entorhinal cortex inputs to the hippocampus for the formation and integration of temporal and contextual memory. Eur J Neurosci 00:1-18. 
Markram H, Muller E, Ramaswamy S, Reimann MW, Abdellah M, Sanchez CA, Ailamaki A, Alonso-Nanclares L, Antille N, Arsever S, Kahou GAA, Berger TK, Bilgili A, Buncic N, Chalimourda A, Chindemi G, Courcol JD, Delalondre F, Delattre V, Druckmann S, et al. (2015) Reconstruction and simulation of neocortical microcircuitry. Cell 163:456-492.

Masliah E, Terry RD, Alford M, Deteresa R (1990) Quantitative immunohistochemistry of synaptophysin in human neocortex: an alternative method to estimate density of presynaptic terminals in paraffin sections. J Histochem Cytochem 38:837844.

Megías M, Emri Z, Freund TF, Gulyás Al (2001) Total number and distribution of inhibitory and excitatory synapses on hippocampal CA1 pyramidal cells. Neuroscience 102:527-540.

Merchán-Pérez A, Rodríguez JR, Alonso-Nanclares L, Schertel A, DeFelipe J (2009) Counting synapses using FIB/SEM microscopy: a true revolution for ultrastructural volume reconstruction. Front Neuroanat 3:18.

Merchán-Pérez A, Rodríguez JR, González S, Robles V, DeFelipe J, Larrañaga P, Bielza C (2014) Three-dimensional spatial distribution of synapses in the neocortex: a dual-beam electron microscopy study. Cereb Cortex 24:1579-1588.

Mikkonen M, Alafuzoff I, Tapiola T, Soininen H, Miettinen R (1999) Subfield- and layer-specific changes in parvalbumin, calretinin and calbindin-D28K immunoreactivity in the entorhinal cortex in Alzheimer's disease. Neuroscience 92:515-532.

Mironov A (2017) Stereological morphometric grids for ImageJ. Ultrastruc Pathol 41:126-126.

Mirra SS, Heyman A, McKeel D, Sumi SM, Crain BJ, Brownlee LM, Vogel FS, Hughes JP, van Belle G, Berg L (1991) The consortium to establish a registry for AD (CERAD). Part II. Standardization of the neuropathologic assessment of AD. Neurology 41:479-486.

Montero-Crespo M, Domínguez-Álvaro $\mathrm{M}$, Rondon-Carrillo $\mathrm{P}$, Alonso-Nanclares L, DeFelipe J, Blazquez-Llorca L (2020) Threedimensional synaptic organization of the human hippocampal CA1 field. Elife 9:e57013.

Montero-Crespo M, Domínguez-Álvaro M, Alonso-Nanclares L, DeFelipe J, Blazquez-Llorca L (2021) Three-dimensional analysis of synaptic organization in the hippocampal CA1 field in Alzheimer's disease. Brain 144:553-573.

Montes J, Peña JM, DeFelipe J, Herreras O, Merchan-Perez A (2015) The influence of synaptic size on AMPA receptor activation: a Monte Carlo model. PLoS One 10:e0130924.

Morales J, Alonso-Nanclares L, Rodríguez JR, DeFelipe J, Rodríguez Á, Merchán- Pérez Á (2011) Espina: a tool for the automated segmentation and counting of synapses in large stacks of electron microscopy images. Front Neuroanat 18:18.

Morales J, Rodríguez A, Rodríguez JR, DeFelipe J, MerchánPérez A (2013) Characterization and extraction of the synaptic apposition surface for synaptic geometric analysis. Front Neuroanat 7:20.

Morrison JH, Baxter MG (2012) The ageing cortical synapse: hallmarks and implications for cognitive decline. Nat Rev Neurosci 13:240-250.

Muramori F, Kobayashi K, Nakamura I (1998) A quantitative study of neurofibrillary tangles, senile plaques and astrocytes in the hippocampal subdivisions and entorhinal cortex in Alzheimer's disease, normal controls and non-Alzheimer neuropsychiatric diseases. Psychiatry Clin Neurosci 52:593-599.

Oorschot D, Peterson D, Jones D (1991) Neurite growth from, and neuronal survival within, cultured explants of the nervous system: a critical review of morphometric and stereological methods, and suggestions for the future. Prog Neurobiol 37:525-546.

Peters A, Palay SL (1996) The morphology of synapses. J Neurocytol 25:687-700.

Peters A, Palay SL, Webster HD (1991) The fine structure of the nervous system: neurons and their supporting cells, pp 70-100. New York: Oxford University Press.
Rajmohan R, Reddy PH (2017) Amyloid-beta and phosphorylated tau accumulations cause abnormalities at synapses of Alzheimer's disease neurons. J Alzheimers Dis 57:975-999.

Rakic P, Bourgeois JP, Goldman-Rakic PS (1994) Synaptic development of the cerebral cortex: implications for learning, memory, and mental illness. Prog Brain Res 102:227-243.

Scheff SW, Price DA (1993) Synapse loss in the temporal lobe in Alzheimer's disease. Ann Neurol 33:190-199.

Scheff SW, Price DA (1998) Synaptic density in the inner molecular layer of the hippocampal dental gyrus in Alzheimer's disease. J Neuropathol Exp Neurol 57:1146-1153.

Scheff SW, Price DA (2003) Synaptic pathology in Alzheimer's disease: a review of ultrastructural studies. Neurobiol Aging 24:10291046.

Scheff SW, Price DA (2006) Alzheimer's disease-related alterations in synaptic density: neocortex and hippocampus. J Alzheimers Dis 9:101-115.

Scheff SW, Dekosky ST, Price DA (1990) Quantitative assessment of cortical synaptic density in Alzheimer's disease. Neurobiol Aging 11:29-37.

Scheff SW, Sparks DL, Price DA (1993) Quantitative assessment of synaptic density in the entorhinal cortex in Alzheimer's disease. Ann Neurol 34:356-361.

Scheff SW, Sparks DL, Price DA (1996) Quantitative assessment of synaptic density in the outer molecular layer of the hippocampal dentate gyrus in Alzheimer's disease. Dementia 7:226232.

Scheff SW, Price DA, Sparks LD (2001) Quantitative assessment of possible age related change in synaptic numbers in the human frontal cortex. Neurobiol Aging 22:355-365.

Scheff SW, Price DA, Schmitt FA, Mufson EJ (2006) Hippocampal synaptic loss in early Alzheimer's disease and mild cognitive impairment. Neurobiol Aging 27:1372-1384.

Scheff SW, Price DA, Schmitt FA, Dekosky ST, Mufson EJ (2007) Synaptic alterations in CA1 in mild Alzheimer disease and mild cognitive impairment. Neurology 68:1501-1508.

Scheff SW, Price DA, Schmitt FA, Scheff MA, Mufson EJ (2011) Synaptic loss in the inferior temporal gyrus in mild cognitive impairment and Alzheimer disease. J Alzheimers Dis 24:547557.

Scheff SW, Price DA, Ansari MA, Roberts KN, Schmitt FA, Ikonomovic MD, Mufson EJ (2015) Synaptic change in the posterior cingulate gyrus in the progression of Alzheimer's disease. $J$ Alzheimers Dis 43:1073-1090.

Schubert D, Kötter R, Staiger JF (2007) Mapping functional connectivity in barrel-related columns reveals layer- and cell type-specific microcircuits. Brain Struct Funct 212:107-119.

Schultz H, Sommer T, Peters J (2015) The role of the human entorhinal cortex in a representational account of memory. Front Hum Neurosci 9:85.

Selkoe DJ (2002) Alzheimer's disease is a synaptic failure. Science 298:789-791.

Sharpe D (2015) Your chi-square test is statistically significant: now what? Pract Assess Res Evaluation 20:8.

Šimić G, Babić Leko M, Wray S, Harrington CR, Delalle I, JovanovMilošević N, Bazõadona D, Buée L, de Silva R, Di Giovanni G, Wischik CM, Hof PR (2017) Monoaminergic neuropathology in Alzheimer's disease. Prog Neurobiol 151:101-138.

Sohal VS, Rubenstein JLR (2019) Excitation-inhibition balance as a framework for investigating mechanisms in neuropsychiatric disorders. Mol Psychiatry 24:1248-1257.

Solodkin A, Van Hoesen GW (1996) Entorhinal cortex modules of the human brain. J Comp Neurol 365:610-617.

Somogyi P, Tamás G, Lujan R, Buhl EH (1998) Salient features of synaptic organisation in the cerebral cortex. Brain Res Brain Res Rev 26:113-135.

Sze CI, Troncoso JC, Kawas C, Mouton P, Price DL, Martin LJ (1997) Loss of the presynaptic vesicle protein synaptophysin in hippocampus correlates with cognitive decline in Alzheimer disease. JNeuropathol Exp Neurol 56:933-944. 
Thal DR, Rüb U, Orantes M, Braak H (2002) Phases of A beta-deposition in the human brain and its relevance for the development of AD. Neurology 58:1791-1800.

Tremblay R, Lee S, Rudy B (2016) GABAergic interneurons in the neocortex: from cellular properties to circuits. Neuron 91:260-292.

Van Hoesen GW, Hyman BT (1990) Hippocampal formation: anatomy and the patterns of pathology in Alzheimer's disease. Prog Brain Res 83:445-457.

Van Hoesen GW, Hyman BT, Damasio AR (1991) Entorhinal cortex pathology in Alzheimer's disease. Hippocampus 1:1-8.

Van Hoesen GW, Augustinack JC, Dierking J, Redman SJ, Thangavel R (2006) The Parahippocampal Gyrus in Alzheimer's Disease: Clinical and Preclinical Neuroanatomical Correlates. Ann NY Acad Sci 911:254-274. von Gunten A, Kövari E, Bussière T, Rivara C-B, Gold G, Bouras C, Hof PR, Giannakopoulos P (2006) Cognitive impact of neuronal pathology in the entorhinal cortex and CA1 field in Alzheimer's disease. Neurobiol Aging 27:270-277.

Xu Y, Zhao M, Han Y, Zhang H (2020) GABAergic inhibitory interneuron deficits in Alzheimer's disease: implications for treatment. Front Neurosci 14:660.

Zhou L, Mclnnes J, Wierda K, Holt M, Herrmann AG, Jackson RJ, Wang YC, Swerts J, Beyens J, Miskiewicz K, Vilain S, Dewachter I, Moechars D, De Strooper B, Spires-Jones TL, De Wit J, Verstreken P (2017) Tau association with synaptic vesicles causes presynaptic dysfunction. Nat Commun 8:15295.

Zhou S, Yu Y (2018) Synaptic E-I balance underlies efficient neural coding. Front Neurosci 12:46. 